\title{
A space-time certified reduced basis method for quasilinear parabolic partial differential equations
}

\author{
Michael Hinze $^{1}$. Denis Korolev ${ }^{1}$ (D)
}

Received: 29 April 2020 / Accepted: 19 March 2021 / Published online: 22 April 2021

(C) The Author(s) 2021

\begin{abstract}
In this paper, we propose a certified reduced basis $(\mathrm{RB})$ method for quasilinear parabolic problems with strongly monotone spatial differential operator. We provide a residualbased a posteriori error estimate for a space-time formulation and the corresponding efficiently computable bound for the certification of the method. We introduce a Petrov-Galerkin finite element discretization of the continuous space-time problem and use it as our reference in a posteriori error control. The Petrov-Galerkin discretization is further approximated by the Crank-Nicolson time-marching problem. It allows to use a POD-Greedy approach to construct the reduced-basis spaces of small dimensions and to apply the Empirical Interpolation Method (EIM) to guarantee the efficient offline-online computational procedure. In our approach, we compute the reduced basis solution in a time-marching framework while the RB approximation error in a space-time norm is controlled by our computable bound. Therefore, we combine a POD-Greedy approximation with a space-time Galerkin method.
\end{abstract}

Keywords Parametrized parabolic equations - Certified reduced basis - Space-time Petrov-Galerkin - A posteriori error estimate $\cdot$ Empirical interpolation method

Mathematics Subject Classification (2010) 35K15 · 35K55 - 65M15 - 65M60

\section{Introduction}

The certified reduced basis method is known as an efficient method for model order reduction of parametrized partial differential equations (see, e.g. [9, 15], where also

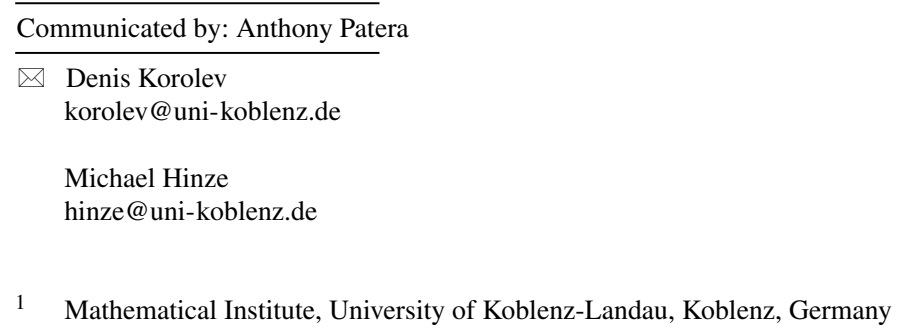


the terminology used in the present article is well-explained). The efficiency comes from the use of the Greedy search algorithm in the basis construction for the numerical approximation of the problem and a posteriori control of the approximation error. The later serves not only for rigorous certification of the method but also as the selection criterion in the Greedy selection process. This process provides incrementally better bases for the approximation and further significant speed-up in multi-query numerical simulations-relevant, for example, in the design, optimization and control contexts, through the use of RB surrogate models.

The reduced basis method was successfully applied to linear [7, 19, 20] and non-linear parabolic problems with polynomial [22, 23] and non-polynomial nonlinearities [5]. In general, there are two approaches for the reduced basis methods applied to unsteady problems: (1) first discretize, then estimate and reduce, (2) first estimate, then discretize and reduce. The approach (1) $[5,7,8]$ is based on a timemarching problem in the offline phase and the error bounds or indicators are then stem from the structure of the discrete problem. The POD-Greedy procedure [8] is commonly used to construct the reduced-basis spaces and the Empirical Interpolation Method (EIM) [3, 6, 14] is used to treat non-affine and non-linear problems [5]. In particular, the approach (1) is applied in [5] to semilinear parabolic problems with monotone non-polynomial non-linearities. However, in this paper, we treat quasilinear parabolic problems with the approach (2). The techniques of [5] in our opinion are not directly applicable to the class of problems we consider. The approach (2) starts from a weak space-time variational formulation (see, e.g. [19, 20, 22, 23]). The error bounds and estimates are then derived in the appropriate Bochner spaces with respect to the natural space-time norms. In this approach, time is treated as a variable and thus it resembles the reduced-basis setting for elliptic problems [16]. The reducedbasis space is consequently constructed in the offline phase out of the space-time snapshots, obtained, for example, with the related Petrov-Galerkin discrete scheme. However, the appropriate choice of the discrete spaces in the Petrov-Galerkin scheme results in a time-marching interpretation (see, e.g. [20, 22]) of the discrete problem. In this way, the time-marching procedure allows to use the standard POD-Greedy approximation and to treat time as the parameter, which leads to the reduced-basis time-marching problem, but the error certification is accomplished with the natural space-time norm error bound. We refer to [4] for the detailed overview and comparison of these two approaches in the context of linear parabolic equations.

We propose an $L^{2}(0, T ; V)$ a posteriori error estimate, based on the spacetime variational formulation of quasilinear parabolic PDEs with strongly monotone differential operators. We introduce a Petrov-Galerkin projection to approximate the continuous variational problem and provide its reduced-basis counterpart. The Petrov-Galerkin problem with its solution $u_{\delta}$ serves as our reference in a posteriori error control. For computational purposes, we approximate the solution $u_{\delta}$ by the solution of the Crank-Nicolson time-marching scheme and consequently use the POD-Greedy procedure to construct the reduced-basis spaces of small dimension. The right-hand side in our error estimate is approximated with a computable bound, which is used in the computational procedure.

The time-marching Crank-Nicolson approximation of the Petrov-Galerkin problem also allows to treat the non-linearity with the EIM in order to have offline-online 
decomposition for our problem available. Moreover, the parameter separability in time, achieved with the EIM, leads to a significant speed-up factor in the computational procedure. The error of the EIM is then also incorporated in the error estimate. We note that on the discrete level, the EIM error is approximated with a computable surrogate and requires one full evaluation of the non-linearity. Hence, the certification procedure is not fully decoupled from operations on a finite-element mesh, compared to standard reduced-basis methods.

Our work is motivated by the structure of the magnetoquasistatic approximation of Maxwell's equations (the eddy current model) and is an extension of the reduced-basis methods for quasilinear PDEs with their applications to magnetostatics problems [11]. This equation finds its place in important applications, such as the computation of magnetic fields in the presence of eddy currents in electrical machines [17]. The development of fast and accurate simulation methods for such problems is of great importance in the optimization and design of electrical machines and other devices [1, 12]. Therefore, there is a demand for reduced order models (see, e.g. [13]) of this quasilinear PDE, which can be further used as surrogates in the optimization procedure. Our approach is applicable to the $2 \mathrm{D}$ magnetoquasistatic problem as well, and we present according numerical results.

\section{Space-time truth solution}

In this section, we consider a space-time variational formulation of quasilinear parabolic partial differential equations, which we denote as the exact problem. The corresponding discrete Petrov-Galerkin approximation is called the truth problem, as it is common in the RB setting. We assume that the solution to the exact problem can be approximated arbitrarily well by the discrete solution of the truth problem. We then neglect the corresponding approximation error.

\subsection{Space-time formulation}

Let $\Omega \subset \mathbb{R}^{d}$ be the spatial domain and $\mu \in \mathcal{D} \subset \mathbb{R}^{p}$, where $\mathcal{D}$ is a compact parameter set. Let $V \subset H^{1}(\Omega)$ be a separable Hilbert space and $H:=$ $L^{2}(\Omega)$. We denote by $\langle\cdot, \cdot\rangle_{V},\langle\cdot, \cdot\rangle_{H}$ and $\|\cdot\|_{V},\|\cdot\|_{H}$ corresponding inner products and induced norms, respectively. To $V$ and $H$, we associate the Gelfand triple $V \hookrightarrow H \hookrightarrow V^{\prime}$ with duality pairing $\langle\cdot, \cdot\rangle_{V^{\prime} V}$. The norm of $l \in V^{\prime}$ is defined by $\|l\|_{V^{\prime}}:=\sup _{\psi \in V,\|\psi\|_{V} \neq 0}\langle l, \psi\rangle_{V^{\prime} V} /\|\psi\|_{V}$. We consider a parametrized quasilinear, bounded differential operator $A: V \times \mathcal{D} \rightarrow V^{\prime}$ with induced quasilinear form

$$
\langle A(u, \mu), v\rangle_{V^{\prime} V}:=a[u](u, v ; \mu)=\int_{\Omega} v(u(x) ; \mu) \nabla u \cdot \nabla v d x,
$$

where the non-linearity satisfies $v(\cdot ; \mu) \in C^{1}(\mathbb{R})$. We assume that the forms (1) are strongly monotone on $V$ with monotonicity constants $m_{a}(\mu)>0$, i.e.

$$
a[v](v, v-w ; \mu)-a[w](w, v-w ; \mu) \geq m_{a}(\mu)\|v-w\|_{V}^{2} \quad \forall v, w \in V,
$$


and Lipschitz continuous on $V$ with Lipschitz constants $L_{a}(\mu)>0$, i.e.

$$
|a[u](u, v ; \mu)-a[w](w, v ; \mu)| \leq L_{a}(\mu)\|u-w\|_{V}\|v\|_{V} \quad \forall u, w, v \in V .
$$

In addition, we assume that these conditions hold uniformly:

$$
m_{a}:=\inf _{\mu \in \mathcal{D}} m_{a}(\mu)>0, \quad L_{a}:=\sup _{\mu \in \mathcal{D}} L_{a}(\mu)<\infty .
$$

For given $\left(g(\cdot ; \mu), u_{o}\right) \in L^{2}\left(I ; V^{\prime}\right) \times H$, we consider the quasilinear parabolic initial value problem of finding $u(t):=u(t ; \mu) \in V, t \in I$ a.e. on the time interval $I=(0, T]$, such that

$$
\dot{u}(t)+A(u(t), \mu)=g(t) \text { in } V^{\prime}, u(0)=u_{o} \text { in } H,
$$

where $\dot{u}:=\frac{\partial u}{\partial t}$ is understood in the generalized sense [23]. We now define a spacetime variational formulation of (5). We use the trial space

$$
\mathcal{X}:=W(0, T)=L^{2}(I ; V) \cap H^{1}\left(I ; V^{\prime}\right)=\left\{v \in L^{2}(I ; V): v, \dot{v} \in L^{2}\left(I ; V^{\prime}\right)\right\}
$$

with the norm $\|w\|_{\mathcal{X}}^{2}:=\|\dot{w}\|_{L^{2}\left(I ; V^{\prime}\right)}^{2}+\|w\|_{L^{2}(I ; V)}^{2}$, and the test space $\mathcal{Y}:=$ $L^{2}(I ; V) \times H$ with the norm $\|v\|_{\mathcal{Y}}^{2}:=\left\|v^{(1)}\right\|_{L^{2}(I ; V)}^{2}+\left\|v^{(2)}\right\|_{H}^{2}$ for $v:=\left(v^{(1)}, v^{(2)}\right)$. The weak formulation of problem (5) reads: find $u:=u(\mu) \in \mathcal{X}$ such that

$$
B[u](u, v ; \mu)=F(v ; \mu), \quad \forall v \in \mathcal{Y},
$$

where

$$
\begin{aligned}
B[u](u, v ; \mu) & :=\int_{I}\left\langle\dot{u}, v^{(1)}\right\rangle_{V^{\prime} V}+a[u]\left(u, v^{(1)} ; \mu\right) d t+\left\langle u(0), v^{(2)}\right\rangle_{H}, \text { and } \\
F(v ; \mu) & :=\int_{I}\left\langle g(\mu), v^{(1)}\right\rangle_{V^{\prime} V} d t+\left\langle u_{o}, v^{(2)}\right\rangle_{H} .
\end{aligned}
$$

Since $\mathcal{X} \hookrightarrow C(I ; H)$, the initial value $u(0)$ is well-defined in $H$ [24]. We note that (2) implies coercivity of the quasilinear form $a[\cdot](\cdot, \cdot ; \mu)$ and (3) implies hemicontinuity, i.e. the continuity of the mapping $s \rightarrow\langle A(u+s w, \mu), v\rangle_{V^{\prime} V}$ for $s \in[0,1]$ and all $u, w, v \in V$. All together, the well-posedness of problem (6) follows, so that (6) admits a unique solution $u \in \mathcal{X}$, see e.g. [25, Theorem 30.A].

\subsection{Petrov-Galerkin truth approximation}

From here onwards, we omit the dependence on $\mu$ wherever appropriate. For the temporal discretization of (6), we use the time grid $0=t^{0}<t^{1}<\ldots<t^{K}=T$ and set $I^{k}=\left(t^{k-1}, t^{k}\right]$ for $k=1, \ldots, K$. We set $\Delta t^{k}=t^{k}-t^{k-1}$ and define $\Delta t:=$ $\max _{1 \leq k \leq K} \triangle t^{k}$. For the spatial discretization, we set $V_{h}=\operatorname{span}\left\{\phi_{1}, \ldots, \phi_{\mathcal{N}_{h}}\right\} \subset V$, where $\operatorname{dim} V_{h}=\mathcal{N}_{h}$ and $h$ denotes the spatial discretization parameter. The functions $\phi_{i}$ will be defined in the numerical examples. With $\delta:=(\triangle t, h)$, we introduce the discrete trial space

$$
\mathcal{X}_{\delta}:=\left\{u_{\delta} \in C^{0}(I ; V),\left.u_{\delta}\right|_{I^{k}} \in \mathcal{P}_{1}\left(I^{k}, V_{h}\right), k=1, \ldots, K\right\} \subset \mathcal{X}
$$


and the discrete test space

$$
\mathcal{Y}_{\delta}:=\left\{v_{\delta} \in L^{2}(I ; V),\left.v_{\delta}\right|_{I^{k}} \in \mathcal{P}_{0}\left(I^{k}, V_{h}\right), k=1, \ldots, K\right\} \times V_{h} \subset \mathcal{Y} .
$$

With these choices of spaces, the truth approximation problem reads: find $u_{\delta}:=$ $u_{\delta}(\mu) \in \mathcal{X}_{\delta}$, such that $u_{\delta}^{0}:=u_{\delta}(0)=P_{H}^{h} u_{o}$ and

$$
B\left[u_{\delta}\right]\left(u_{\delta}, v_{\delta} ; \mu\right)=F\left(v_{\delta} ; \mu\right) \quad \forall v_{\delta} \in \mathcal{Y}_{\delta},
$$

where $P_{H}^{h}: H \rightarrow V_{h}$ denotes the $H$-orthogonal projection onto $V_{h}$. It follows as for (6) that problem (9) admits a unique solution $u_{\delta} \in \mathcal{X}_{\delta}$.

The Petrov-Galerkin space-time discrete formulation (9) is approximated by the Crank-Nicolson time-stepping scheme. Indeed, since the test space $\mathcal{Y}_{\delta}$ consists of piecewise constant polynomials in time, the problem can be solved via the following procedure for $k=1, \ldots, K$ :

$$
\int_{I^{k}}\left\langle\dot{u}_{\delta}, v_{h}\right\rangle_{V^{\prime} V}+a\left[u_{\delta}\right]\left(u_{\delta}, v_{h} ; \mu\right) d t=\int_{I^{k}}\left\langle g(\mu), v_{h}\right\rangle_{V^{\prime} V} d t \quad \forall v_{h} \in V_{h} .
$$

Since the trial space $\mathcal{X}_{\delta}$ consists of piecewise linear and continuous polynomials in time with the values $\hat{u}_{\delta}^{k}:=u_{\delta}\left(t^{k}\right)$ and $\hat{u}_{\delta}^{k-1}:=u_{\delta}\left(t^{k-1}\right)$, we can represent $u_{\delta}$ on $I^{k}$ as the linear function

$$
u_{\delta}(t)=\frac{1}{\Delta t^{k}}\left\{\left(t^{k}-t\right) \hat{u}_{\delta}^{k-1}+\left(t-t^{k-1}\right) \hat{u}_{\delta}^{k}\right\}, t \in I^{k} .
$$

We use the representation (11) in (10), test (10) against the basis functions $\phi_{i} \in$ $V_{h}\left(i=1, \ldots, \mathcal{N}_{h}\right)$ and use the trapezoidal quadrature rule for the approximation of the appearing integrals. In this way, we obtain the Crank-Nicolson time-stepping scheme, which for $k=1, \ldots, K$ reads

$$
\begin{array}{r}
\left\langle u_{\delta}^{k}-u_{\delta}^{k-1}, \phi_{i}\right\rangle_{H}+\frac{\Delta t^{k}}{2}\left\{a\left[u_{\delta}^{k}\right]\left(u_{\delta}^{k}, \phi_{i} ; \mu\right)+a\left[u_{\delta}^{k-1}\right]\left(u_{\delta}^{k-1}, \phi_{i} ; \mu\right)\right\}= \\
=\frac{\Delta t^{k}}{2}\left\{\left\langle g\left(t^{k} ; \mu\right), \phi_{i}\right\rangle_{V^{\prime} V}+\left\langle g\left(t^{k-1} ; \mu\right), \phi_{i}\right\rangle_{V^{\prime} V}\right\}, \quad 1 \leq i \leq \mathcal{N}_{h},
\end{array}
$$

where we consider $u_{\delta}^{k}$ as our approximation of $\hat{u}_{\delta}^{k}$. Here, we recall that the initial condition $u_{\delta}^{0}$ is obtained as an $H$-orthogonal projection of $u_{o}$ onto $V_{h}$. Given the ansatz $u_{\delta}^{k}=\sum_{i=1}^{\mathcal{N}_{h}} u_{i}^{k} \phi_{i}$ and defining $\mathbf{u}_{\delta}^{k}:=\left\{u_{i}^{k}\right\}_{i=1}^{\mathcal{N}_{h}} \in \mathbb{R}^{\mathcal{N}_{h}}$, the resulting non-linear algebraic equations are then solved by applying Newton's method for finding the root $\mathbf{u}_{\delta}^{k}$ of

$$
\begin{aligned}
\mathbf{G}_{h}\left(\mathbf{u}_{\delta}^{k} ; \mu\right):= & \frac{1}{\Delta t^{k}} \mathbf{M}_{h}\left(\mathbf{u}_{\delta}^{k}-\mathbf{u}_{\delta}^{k-1}\right)-\frac{1}{2}\left[\mathbf{g}_{h}^{k}(\mu)+\mathbf{g}_{h}^{k-1}(\mu)\right] \\
& +\frac{1}{2}\left[\mathbf{A}_{h}\left(\mathbf{u}_{\delta}^{k} ; \mu\right) \mathbf{u}_{\delta}^{k}+\mathbf{A}_{h}\left(\mathbf{u}_{\delta}^{k-1} ; \mu\right) \mathbf{u}_{\delta}^{k-1}\right],
\end{aligned}
$$

where $\mathbf{M}_{h}:=\left\{\left\langle\phi_{i}, \phi_{j}\right\rangle_{H}\right\}_{i, j=1}^{\mathcal{N}_{h}}, \mathbf{A}_{h}\left(\mathbf{u}_{\delta}^{k} ; \mu\right):=\left\{a\left[u_{\delta}^{k}\right]\left(\phi_{i}, \phi_{j} ; \mu\right)\right\}_{i, j=1}^{\mathcal{N}_{h}} \in \mathbb{R}^{\mathcal{N}_{h} \times \mathcal{N}_{h}}$ and $\mathbf{g}_{h}^{k}(\mu):=\left\{\left\langle g\left(t^{k} ; \mu\right), \phi_{i}\right\rangle_{V^{\prime} V}\right\}_{i=1}^{\mathcal{N}_{h}} \in \mathbb{R}^{\mathcal{N}_{h}}$. The initial condition for (13) is given by $\mathbf{u}_{\delta}^{0}:=\left\{\left\langle u_{o}, \phi_{i}\right\rangle_{H}\right\}_{i=1}^{\mathcal{N}_{h}} \in \mathbb{R}^{\mathcal{N}_{h}}$. The strong monotonicity of the quasilinear form (2) guarantees that (13) admits a unique root $\mathbf{u}_{\delta}^{k}$ for every parameter $\mu \in \mathcal{D}$. 
Newton's iteration for finding a root of (13) reads: starting with $\mathbf{u}_{\delta}^{k,(0)}$, for $z=$ $0,1, \ldots$ solve the linear system

$$
\mathbf{J}_{h}\left(\mathbf{u}_{\delta}^{k,(z)} ; \mu\right) \delta \mathbf{u}_{\delta}^{k,(z)}=-\mathbf{G}_{h}\left(\mathbf{u}_{\delta}^{k,(z)} ; \mu\right)
$$

to obtain $\delta \mathbf{u}_{\delta}^{k,(z)}$, and then update the solution $\mathbf{u}_{\delta}^{k,(z+1)}:=\mathbf{u}_{\delta}^{k,(z)}+\delta \mathbf{u}_{\delta}^{k,(z)}$. The system Jacobian matrix is given by

$$
\mathbf{J}_{h}\left(\mathbf{u}_{\delta}^{k} ; \mu\right)=\frac{1}{\Delta t^{k}} \mathbf{M}_{h}+\frac{1}{2} \mathbf{A}_{h}^{\prime}\left(\mathbf{u}_{\delta}^{k} ; \mu\right)
$$

where $\mathbf{A}_{h}^{\prime}\left(\mathbf{u}_{\delta}^{k} ; \mu\right):=\left\{d a\left[u_{\delta}^{k}\right]\left(\phi_{i}, \phi_{j} ; \mu\right)\right\}_{i, j=1}^{\mathcal{N}_{h}} \in \mathbb{R}^{\mathcal{N}_{h} \times \mathcal{N}_{h}}$. Here, we assume the existence of the Fréchet derivative $A^{\prime}(u ; \mu): V \times \mathcal{D} \rightarrow V^{\prime}$ of the non-linear operator $A(u ; \mu)$ for every parameter $\mu \in \mathcal{D}$, which induces the corresponding bilinear form $\left\langle A^{\prime}(u ; \mu) v, w\right\rangle_{V^{\prime} V}=d a[u](v, w ; \mu)$. We will specify it later for our examples. We note that $\mathbf{A}_{h}^{\prime}\left(\mathbf{u}_{\delta}^{k} ; \mu\right)$ is positive definite, since $d a[u](\cdot, \cdot ; \mu)$ is coercive due to the strong monotonicity of $A$; therefore, the system (14) admits a unique solution.

\section{The reduced basis method}

In this section, we introduce the reduced basis model and its numerical realization. Then, we introduce our a posteriori error bound and discuss its efficient evaluation.

\subsection{Empirical interpolation of the non-linearity}

We use the Empirical Interpolation Method (EIM) [3] to ensure the availability of an affine decomposition for the quasilinear form $a\left[u_{\delta}^{k}\right](\cdot, \cdot ; \mu)$ for every parameter $\mu \in \mathcal{D}$. We then need to find a parameter-separable (affine) counterpart $v_{M}(\cdot ; \mu)$ of the non-linear non-affine function $v(\cdot ; \mu)$. For EIM non-linearity approximation, we treat time as an additional parameter in the problem; thus, we set $\mathbb{I}:=$ $\{1, \ldots, K\}$ as our discrete time set. Given an EIM tolerance $\epsilon_{E I M}>0$ and a fine sample $\mathcal{D}_{\text {train }}^{E I M} \subset \mathcal{D}$ of size $n_{\text {train }}^{\text {EIM }}$, we construct with Algorithm 1 the nested sample sets $S_{M}^{v} \subset \mathcal{D}_{\text {train }}^{E I M}$ and $\mathbb{I}_{M}^{v} \subset \mathbb{I}$, where $S_{M}^{v}:=\left\{\mu_{1}^{v} \in \mathcal{D}_{\text {train }}^{\text {EIM }}, \ldots, \mu_{M}^{v} \in\right.$ $\left.\mathcal{D}_{\text {train }}^{\text {EIM }}\right\}$ and $\mathbb{I}_{M}^{v}:=\left\{k_{1}^{M} \in \mathbb{I}, \ldots, k_{M}^{M} \in \mathbb{I}\right\}$, and associated approximation spaces $W_{M}^{v}:=\operatorname{span}\left\{v\left(u_{\delta}^{k_{m}^{M}}\left(\cdot ; \mu_{m}^{v}\right) ; \mu_{m}^{v}\right), 1 \leq m \leq M\right\}=\operatorname{span}\left\{q_{1}, \ldots, q_{M}\right\}$. Algorithm 1 also provides the nested sets of interpolation points $T_{M}=\left\{x_{1}^{M}, \ldots, x_{M}^{M}\right\}, \quad 1 \leq$ $M \leq M_{\max }$. We build an affine approximation $v_{M}\left(u_{\delta}^{k}(x) ; \mu\right)$ of $v\left(u_{\delta}^{k}(x) ; \mu\right)$ for our time-marching scheme according to

$$
\begin{aligned}
v_{M}\left(u_{\delta}^{k}(x) ; \mu\right) & :=\sum_{m=1}^{M} \varphi_{m}^{k}(\mu) q_{m}(x) \\
& =\sum_{m=1}^{M}\left(\mathbf{B}_{M}^{-1} v_{\mu}^{k}\right)_{m} q_{m}(\hat{x})=v\left(u_{\delta}^{k}(x) ; \mu\right)+\epsilon_{M}^{k}(x ; \mu),
\end{aligned}
$$


where $\epsilon_{M}^{k}(\cdot ; \mu)$ is the EIM approximation error: it holds $\left\|\epsilon_{M}^{k}(\cdot ; \mu)\right\|_{L^{\infty}(\Omega)}<\epsilon_{E I M}$ for all $k \in \mathbb{I}, \mu \in \mathcal{D}_{\text {train }}^{\text {EIM }}$. In (16), we also have $v_{\mu}^{k}:=\left\{v\left(u_{\delta}^{k}\left(x_{m}^{M}\right) ; \mu\right)\right\}_{m=1}^{M} \in \mathbb{R}^{M}$ and $\mathbf{B}_{M} \in \mathbb{R}^{M \times M}$ is the lower triangular interpolation matrix $\left(\mathbf{B}_{M}\right)_{i j}=q_{j}\left(x_{i}\right)$ with $\left(\mathbf{B}_{M}\right)_{i i}=1(i=1, \ldots, M)$ by construction.

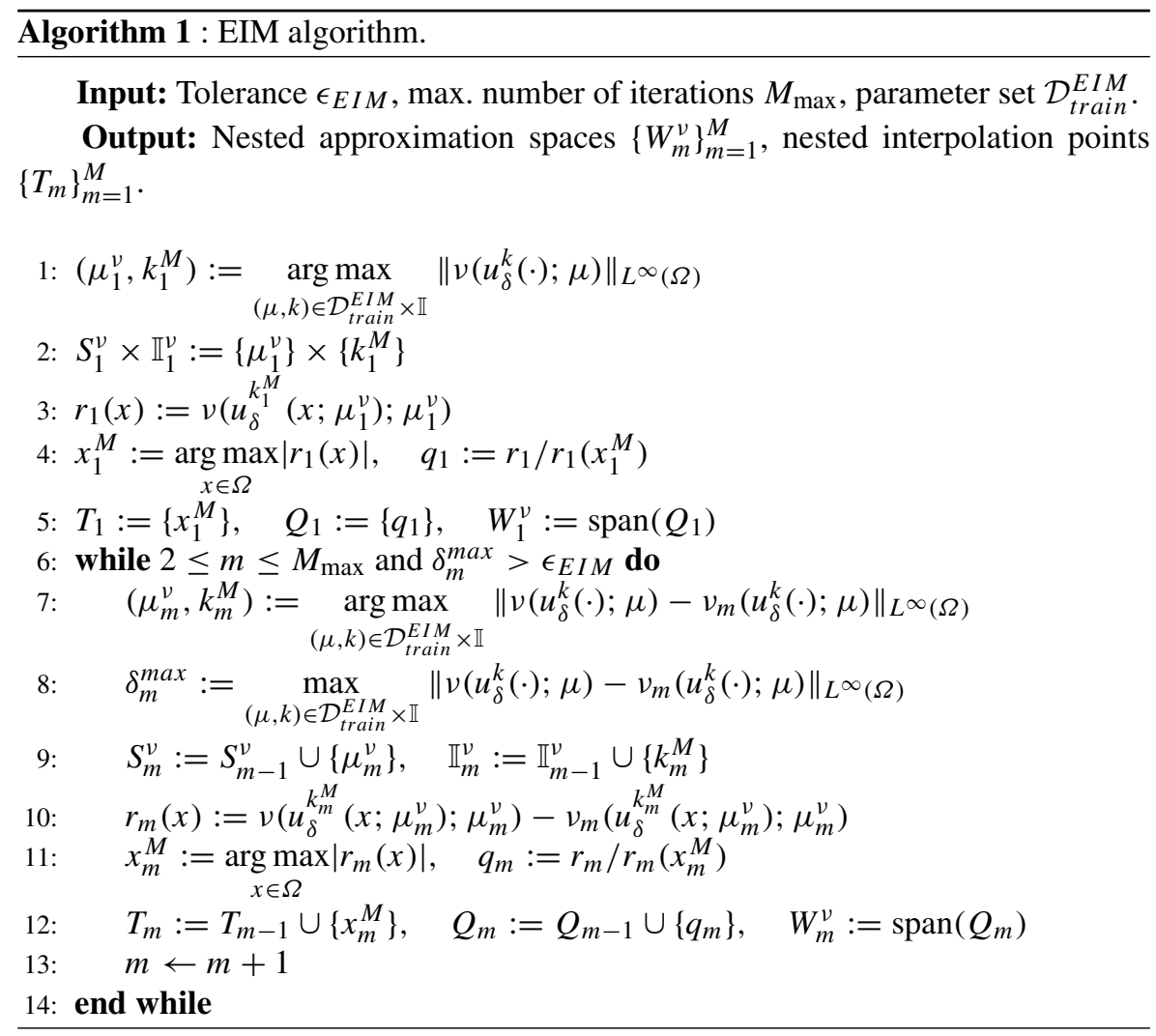

We then have the EIM approximation $\tilde{a}[\cdot](\cdot, \cdot ; \mu)$ of the quasilinear form $a[\cdot](\cdot, \cdot ; \mu)$, which admits the affine decomposition

$$
\tilde{a}\left[u_{\delta}^{k}\right]\left(u_{\delta}^{k}, v ; \mu\right)=\sum_{m=1}^{M} \varphi_{m}^{k}(\mu) \tilde{a}_{m}\left(u_{\delta}^{k}, v\right), \quad \tilde{a}_{m}\left(u_{\delta}^{k}, v\right)=\int_{\Omega} q_{m} \nabla u_{\delta}^{k} \cdot \nabla v d x .
$$

For mathematical convenience, we assume that the EIM approximation $\tilde{a}[\cdot](\cdot, \cdot ; \mu)$ is sufficiently accurate in the sense that the form $\tilde{a}[\cdot](\cdot, \cdot ; \mu)$ is strongly monotone on $V_{h}$ with monotonicity constant $\tilde{m}_{a}(\mu):=m_{a}(\mu) \pm \epsilon_{a}>0$, i.e. for all $v_{h}, w_{h} \in V_{h}$ it holds

$$
\tilde{a}\left[v_{h}\right]\left(v_{h}, v_{h}-w_{h} ; \mu\right)-\tilde{a}\left[w_{h}\right]\left(w_{h}, v_{h}-w_{h} ; \mu\right) \geq \tilde{m}_{a}(\mu)\left\|v_{h}-w_{h}\right\|_{V}^{2},
$$


and Lipschitz continuous on $V_{h}$ with Lipschitz constants $\tilde{L}_{a}(\mu):=L_{a}(\mu) \pm \epsilon_{a}>0$, i.e. for all $u_{h}, w_{h}, v_{h} \in V_{h}$ it holds

$$
\left|\tilde{a}\left[u_{h}\right]\left(u_{h}, v_{h} ; \mu\right)-\tilde{a}\left[w_{h}\right]\left(w_{h}, v_{h} ; \mu\right)\right| \leq \tilde{L}_{a}(\mu)\left\|u_{h}-w_{h}\right\|_{V}\left\|v_{h}\right\|_{V},
$$

where $\epsilon_{a} \in \mathbb{R}_{+}$is small enough and is related to the EIM approximation error. However, in the EIM practice, we can guarantee that conditions (18) and (19) hold uniformly together with $\epsilon_{a}<\epsilon_{E I M}$ only on $\mathcal{D}_{\text {train }}^{E I M}$. It is difficult to check these properties a priori for $\mu \in \mathcal{D} \backslash \mathcal{D}_{\text {train }}^{\text {EIM }}$, so that arguing the well-posedness of the upcoming discrete systems (23) and (24) in general is not possible.

We also assume the affine decomposition

$$
\left\langle g\left(t^{k} ; \mu\right), v\right\rangle_{V^{\prime} V}=\sum_{q=1}^{Q_{g}} \theta_{g, q}^{k}(\mu)\left\langle g_{q}, v\right\rangle_{V^{\prime} V}
$$

for the right-hand side, where $\theta_{g, q}^{k}: \mathcal{D} \rightarrow \mathbb{R}$ are parameter-dependent functions and parameter-independent forms $g_{q}: V \rightarrow \mathbb{R}, k=1, \ldots, K, q=1, \ldots, Q_{g}$. If (20) is not available, the EIM procedure can be similarly applied.

\subsection{Reduced basis approximation with the POD-Greedy method}

The idea of the reduced-basis approximation consists in replacing the "truth" (highdimensional) space $V_{h}$ in the definition of $\mathcal{X}_{\delta}$ and $\mathcal{Y}_{\delta}$ by a low-dimensional subspace $V_{N} \subset V_{h}$. With $V_{N}$ available, we introduce the corresponding reduced trial space

$$
\mathcal{X}_{\triangle t, N}:=\left\{u_{N} \in C^{0}(I ; V),\left.u_{N}\right|_{I^{k}} \in \mathcal{P}_{1}\left(I^{k}, V_{N}\right), k=1, \ldots, K\right\}
$$

and the reduced test space

$$
\mathcal{Y}_{\triangle t, N}:=\left\{v_{N} \in L^{2}(I ; V),\left.v_{N}\right|_{I^{k}} \in \mathcal{P}_{0}\left(I^{k}, V_{N}\right), k=1, \ldots, K\right\} \times V_{N}
$$

We construct $V_{N}:=\operatorname{span}\left\{\xi_{1}, \ldots, \xi_{N}\right\} \subset V_{h}$ by the POD-Greedy procedure in Algorithm 2, compare, e.g. [8]. We denote by $\mathcal{D}_{\text {train }} \subset \mathcal{D}$ a fine sample of size $n_{\text {train }}$. In our setting, the POD-Greedy algorithm constructs iteratively nested spaces $V_{n}, 1 \leq n \leq N$ using an a posteriori error estimator $\triangle(Y ; \mu)$ (see the next section for details on a posteriori error analysis), which predicts the expected approximation error for a given parameter $\mu \in \mathcal{D}_{\text {train }}$ in the space $Y:=\mathcal{Y}_{\triangle t, n}$. We want the expected approximation error to be less than the prescribed tolerance $\varepsilon_{R B}>0$. We initiate the algorithm with the choice of the initial basis vector $\xi_{1}:=u_{\delta}^{0} /\left\|u_{\delta}^{0}\right\|_{V}$; this choice is motivated by the assumption in Proposition 1. The snapshots $u_{\delta}^{k}(\mu)$ for the procedure are provided by the Crank-Nicolson scheme (12). Next, we proceed as stated in the following Algorithm 2. 


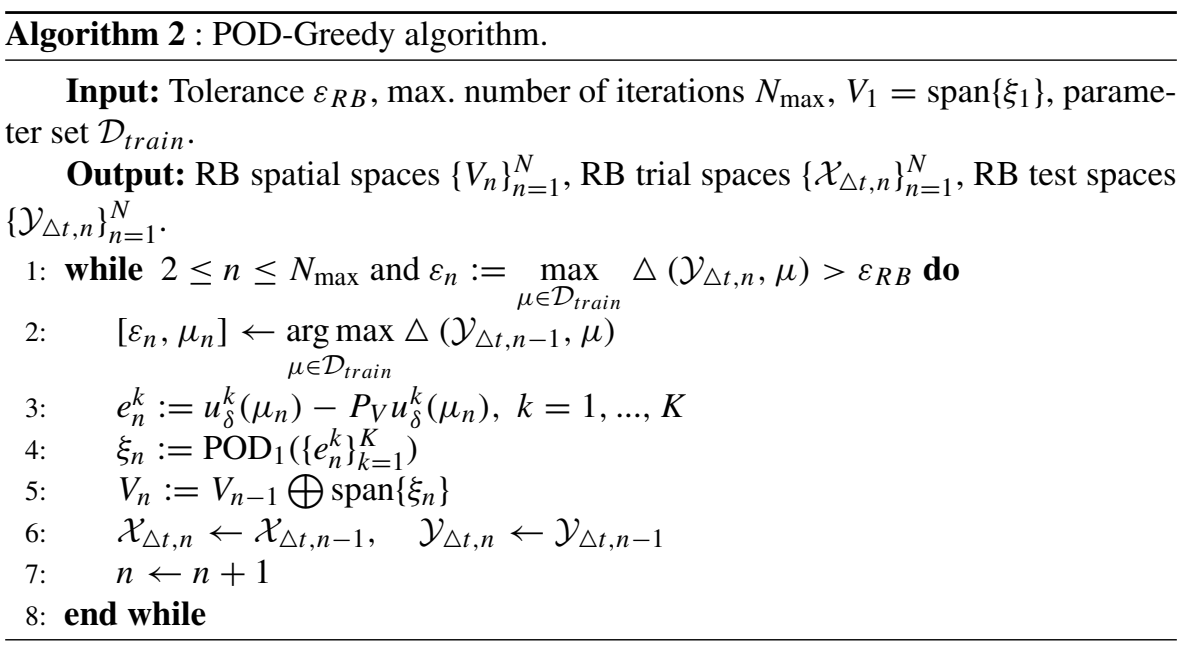

In Algorithm 2, $P_{V}: V_{h} \rightarrow V_{n}$ denotes the $V$-orthogonal projection, and the operation $\operatorname{POD}_{1}\left(\left\{e_{n}^{k}\right\}_{k=1}^{K}\right)$ denotes the extraction of the dominant mode of the Proper Orthogonal Decomposition (see, e.g. [21]). We also note that more modes could be extracted in every step of the algorithm: it reduces the offline computational time, but the produced reduced-basis space will be of larger dimension in general, compared to the approach, which we use here.

The reduced-basis approximation of problem (9) reads: find $u_{N}:=u_{N}(\mu) \in$ $\mathcal{X}_{\triangle t, N}$, such that $u_{N}^{0}:=u_{N}(0)=P_{H}^{N} u_{o}$ and

$$
\tilde{B}\left[u_{N}\right]\left(u_{N}, v_{N} ; \mu\right)=\tilde{F}\left(v_{N} ; \mu\right) \quad \forall v_{N} \in \mathcal{Y}_{\triangle t, N},
$$

where

$$
\begin{gathered}
\tilde{B}\left[u_{N}\right]\left(u_{N}, v_{N} ; \mu\right)=\int_{I}\left\langle\dot{u}_{N}, v_{N}^{(1)}\right\rangle_{V^{\prime} V}+\tilde{a}\left[u_{N}\right]\left(u_{N}, v_{N}^{(1)} ; \mu\right) d t+\left\langle P_{H}^{N} u_{o}, v_{N}^{(2)}\right\rangle_{H}, \\
\tilde{F}\left(v_{N} ; \mu\right): \\
=\int_{I}\left\langle g(\mu), v_{N}^{(1)}\right\rangle_{V^{\prime} V} d t+\left\langle u_{\delta}^{0}, v_{N}^{(2)}\right\rangle_{H},
\end{gathered}
$$

and $P_{H}^{N}: V_{h} \rightarrow V_{N}$ denotes the $H$-orthogonal projection onto $V_{N}$. It follows as for Eq. 6 from our assumptions (18) and (19) that problem (21) admits a unique solution $u_{N}(\mu) \in \mathcal{X}_{\triangle t, N}$ for all $\mu \in \mathcal{D}$.

Problem (21) is approximated by the reduced-basis Crank-Nicolson timemarching scheme with the EIM approximation of the non-linearity, i.e.

$$
\begin{aligned}
\left\langle u_{N}^{k}-u_{N}^{k-1}, v_{N}^{(1)}\right\rangle_{H} & +\frac{\Delta t^{k}}{2}\left\{\tilde{a}\left[u_{N}^{k}\right]\left(u_{N}^{k}, v_{N}^{(1)} ; \mu\right)+\tilde{a}\left[u_{N}^{k-1}\right]\left(u_{N}^{k-1}, v_{N}^{(1)} ; \mu\right)\right\} \\
& =\frac{\Delta t^{k}}{2}\left\{\left\langle g\left(t^{k} ; \mu\right), v_{N}^{(1)}\right\rangle_{V^{\prime} V}+\left\langle g\left(t^{k-1} ; \mu\right), v_{N}^{(1)}\right\rangle_{V^{\prime} V}\right\},
\end{aligned}
$$

where the initial condition $u_{N}^{0}$ is obtained as an $H$-projection of $u_{\delta}^{0}$ onto $V_{N}$. The resulting non-linear algebraic equations are then solved with the RB counterpart of 
Newton's method by finding the root of

$$
\begin{aligned}
\mathbf{G}_{N, M}\left(\mathbf{u}_{N}^{k} ; \mu\right) & =\frac{1}{\triangle t^{k}} \mathbf{M}_{N}\left(\mathbf{u}_{N}^{k}-\mathbf{u}_{N}^{k-1}\right)-\frac{1}{2}\left[\mathbf{g}_{N}^{k}(\mu)+\mathbf{g}_{N}^{k-1}(\mu)\right] \\
& +\frac{1}{2}\left[\mathbf{A}_{N, M}\left(\mathbf{u}_{N}^{k} ; \mu\right) \mathbf{u}_{N}^{k}+\mathbf{A}_{N, M}\left(\mathbf{u}_{N}^{k-1} ; \mu\right) \mathbf{u}_{N}^{k-1}\right],
\end{aligned}
$$

where $\mathbf{M}_{N}:=\left\{\left\langle\xi_{i}, \xi_{j}\right\rangle_{H}\right\}_{i, j=1}^{N}, \mathbf{A}_{N, M}\left(\mathbf{u}_{N}^{k} ; \mu\right):=\left\{\tilde{a}\left[u_{N}^{k}\right]\left(\xi_{i}, \xi_{j} ; \mu\right)\right\}_{i, j=1}^{N} \in \mathbb{R}^{N \times N}$ and $\mathbf{g}_{N}^{k}(\mu):=\left\{\left\langle g\left(t^{k} ; \mu\right), \xi_{i}\right\rangle_{V^{\prime} V}\right\}_{i=1}^{N} \in \mathbb{R}^{N}$. The initial condition is given by $\mathbf{u}_{N}^{0}:=\left\{\left\langle u_{\delta}^{0}, \xi_{i}\right\rangle_{H}\right\}_{i=1}^{N} \in \mathbb{R}^{N}$. The strong monotonicity (18) of the quasilinear form (17) guarantees that (23) admits a unique root $\mathbf{u}_{N}^{k}$ for every parameter $\mu \in \mathcal{D}$. We remind that the strong monotonicity property (18) is based on the assumption about the accuracy of EIM.

The Newton's iteration for finding a root of (23) reads: starting with $\mathbf{u}_{N}^{k,(0)}$, for $z=0,1, \ldots$ solve the linear system

$$
\mathbf{J}_{N, M}\left(\mathbf{u}_{N}^{k,(z)} ; \mu\right) \delta \mathbf{u}_{N}^{k,(z)}=-\mathbf{G}_{N, M}\left(\mathbf{u}_{N}^{k,(z)} ; \mu\right)
$$

to obtain $\delta \mathbf{u}_{N}^{k,(z)}$, and then update the solution $\mathbf{u}_{N}^{k,(z+1)}:=\mathbf{u}_{N}^{k,(z)}+\delta \mathbf{u}_{N}^{k,(z)}$. The system Jacobian matrix is given by

$$
\mathbf{J}_{N, M}\left(\mathbf{u}_{N}^{k} ; \mu\right)=\frac{1}{\Delta t^{k}} \mathbf{M}_{N}+\frac{1}{2} \mathbf{A}_{N, M}^{\prime}\left(\mathbf{u}_{N}^{k} ; \mu\right) .
$$

If the mapping $\mu \mapsto \mathbf{A}_{N, M}^{\prime}(\cdot ; \mu)$ is bounded in $\mu \in \mathcal{D}$, then for $\Delta t^{k} \leq C(\mathcal{D})$, where $C(\mathcal{D})>0$ is some constant, the Jacobian matrix $(25)$ is invertible.

We will comment on the computation of the reduced parametrized counterpart $\mathbf{A}_{N, M}^{\prime}\left(\mathbf{u}_{N}^{k} ; \mu\right):=\left\{d \tilde{a}\left[u_{N}^{k}\right]\left(\xi_{i}, \xi_{j} ; \mu\right)\right\}_{i, j=1}^{N} \in \mathbb{R}^{N \times N}$ of $\mathbf{A}_{h}^{\prime}\left(\mathbf{u}_{\delta}^{k} ; \mu\right)$ in (25). We have

$$
\tilde{a}\left[u_{N}^{k}\right]\left(u_{N}, \xi_{i} ; \mu\right)=\sum_{j=1}^{N} \sum_{m=1}^{M} \varphi_{m}^{k}(\mu) \tilde{a}_{m}\left(\xi_{j}, \xi_{i}\right) u_{N, j}^{k}, \quad 1 \leq i \leq N .
$$

With the EIM approximation of the non-linearity, it follows that

$$
\begin{aligned}
\sum_{s=1}^{M}\left(\mathbf{B}_{M}\right)_{m, s} \varphi_{m, s}^{k}(\mu) & =v\left(u_{N}^{k}\left(x_{m}^{M} ; \mu\right) ; \mu\right), \quad 1 \leq m \leq M \\
& =v\left(\sum_{n=1}^{N} u_{N, n}^{k} \xi_{n}\left(x_{m}^{M}\right) ; \mu\right), \quad 1 \leq m \leq M .
\end{aligned}
$$

Plugging (27) into (26) results in

$$
\tilde{a}\left[u_{N}^{k}\right]\left(u_{N}, \xi_{i} ; \mu\right)=\sum_{j=1}^{N} \sum_{m=1}^{M} \mathbf{D}_{i, m}^{N, M}\left(\mathbf{u}_{N}^{k} ; \mu\right) v\left(\sum_{n=1}^{N} u_{N, n}^{k} \xi_{n}\left(x_{m}^{M}\right) ; \mu\right) u_{N, j}^{k}
$$


with $\mathbf{D}^{N, M}\left(\mathbf{u}_{N}^{k} ; \mu\right)=\mathbf{A}_{N, M}\left(\mathbf{u}_{N}^{k} ; \mu\right)\left(\mathbf{B}_{M}\right)^{-1} \in \mathbb{R}^{N \times M}$. Taking the derivative of (28) with respect to the components $u_{N, j}^{k}(\mu), 1 \leq j \leq N$, we derive the formula for $\mathbf{A}_{N, M}^{\prime}\left(\mathbf{u}_{N}^{k} ; \mu\right)=\mathbf{A}_{N, M}\left(\mathbf{u}_{N}^{k} ; \mu\right)+\mathbf{E}_{N, M}\left(\mathbf{u}_{N}^{k} ; \mu\right)$, where

$$
\left(\mathbf{E}_{N, M}\left(\mathbf{u}_{N}^{k} ; \mu\right)\right)_{i, j}=\sum_{s=1}^{N} u_{N, s}^{k} \sum_{m=1}^{M} \mathbf{D}_{i, m}^{N, M}\left(\mathbf{u}_{N}^{k} ; \mu\right) \frac{\partial}{\partial u_{N, j}^{k}} v\left(u_{N}^{k}\left(x_{m}^{M}\right) ; \mu\right)
$$

We will give the exact form of $\frac{\partial}{\partial u_{N, j}^{k}} v\left(u_{N}^{k}\left(x_{m}^{M}\right) ; \mu\right)$ in the upcoming examples. We note that a more complicated formula for (29) can be obtained in the presence of geometry parametrisation [11].

The proposed reduced numerical scheme contains parameter-separable matrices and thus allows offline-online decomposition. The offline phase (model construction) depends on expensive high-dimensional finite element simulations and thus on $\mathcal{N}$, but should be performed only once. However, the assembling of all the highdimensional parameter-dependent quantities is computationally simplified due to the affine dependence on the parameters (17),(20). In the online phase (RB model simulation), the computational complexity scales polynomially in $N$ and $M$, independently of $\mathcal{N}$ and thus is inexpensive. The operation count associated with each Newton update of the residual $\mathbf{G}_{N, M}\left(\mathbf{u}_{N}^{k,(z)}\right)$ in the online phase is $\mathcal{O}\left(N^{2} Q_{a}+N^{2}+\right.$ $\left.M^{2}+N Q_{f_{o}}\right)$ and the Jacobian $\mathbf{J}_{N, M}\left(\mathbf{u}_{N, M}^{k,(z)}\right)$ is assembled at cost $\mathcal{O}\left(M N^{3}\right)$ with the dominant cost of assembling $\mathbf{E}_{N, M}\left(\mathbf{u}_{N}^{k} ; \mu\right)$, and then inverted at cost $\mathcal{O}\left(N^{3}\right)$.

\subsection{Reduced basis certification}

An important ingredient of the reduced basis methodology is the verification of the error (certification of the reduced basis method). In the present work, we provide a residual-based a posteriori error estimate. We denote by $R(\cdot ; \mu) \in \mathcal{Y}_{\delta}^{\prime}$ the residual of the problem, defined naturally as:

$$
R\left(v_{\delta} ; \mu\right):=F\left(v_{\delta} ; \mu\right)-\tilde{B}\left[u_{N}\right]\left(u_{N}, v_{\delta} ; \mu\right)=\int_{I}\left\langle r(t ; \mu), v_{\delta}\right\rangle_{V^{\prime} V} d t \quad \forall v_{\delta} \in \mathcal{Y}_{\delta}
$$

We have the following

Proposition 1 (A posteriori Error Estimate) Let $m_{a}(\mu)>0$ be a monotonicity constant from (2) and assume that $u_{\delta}^{0} \in V_{N}$. Then, the error $e(\mu)=u_{\delta}(\mu)-u_{N}(\mu)$ of the reduced basis approximation is bounded by

$$
\|e(\mu)\| \mathcal{Y} \leq \frac{1}{m_{a}(\mu)}\left(\|R(\cdot ; \mu)\|_{\mathcal{Y}_{\delta}^{\prime}}+\delta_{M}(\mu)\left\|u_{N}(\mu)\right\|_{L^{2}(I ; V)}\right)=: \triangle_{N, M}^{c}(\mu),
$$

where

$$
\delta_{M}(\mu)=\sup _{t \in I} \sup _{x \in \Omega}\left|v_{M}\left(u_{N}(x, t) ; \mu\right)-v\left(u_{N}(x, t) ; \mu\right)\right|
$$

denotes the approximation error of the non-linearity. 
Proof Since in the case $e=0$ there is nothing to show, we assume that $e \neq 0$. We have $u_{\delta}^{0} \in V_{N}$ and $\left.P_{H}^{N}\right|_{V_{N}}=I d$; therefore, $u_{N}^{0}:=P_{H}^{N} u_{\delta}^{0}=u_{\delta}^{0}$. It implies that $\|e(0)\|_{H}=0,\|e\|_{\mathcal{Y}}=\|e\|_{L^{2}(I ; V)}$ and $\|R(\cdot ; \mu)\|_{\mathcal{Y}_{\delta}^{\prime}}=\|R(\cdot ; \mu)\|_{L^{2}\left(I ; V_{h}^{\prime}\right)}$. First, we obtain the following estimate by applying Cauchy-Schwartz inequality:

$$
\begin{array}{r}
\tilde{a}\left[u_{N}\right]\left(u_{N}, e ; \mu\right)-a\left[u_{N}\right]\left(u_{N}, e ; \mu\right)=\int_{\Omega}\left[v_{M}\left(u_{N} ; \mu\right)-v\left(u_{N} ; \mu\right)\right] \nabla u_{N} \cdot \nabla e d x(33) \\
\leq \sup _{x \in \Omega}\left|v_{M}\left(u_{N}(x, \cdot) ; \mu\right)-v\left(u_{N}(x, \cdot) ; \mu\right)\right|\left\|u_{N}\right\|_{V}\|e\|_{V} .
\end{array}
$$

Integrating (33) in $t$ and applying the Cauchy-Schwartz inequality to the corresponding integral, we get:

$$
\int_{I} \tilde{a}\left[u_{N}\right]\left(u_{N}, e ; \mu\right)-a\left[u_{N}\right]\left(u_{N}, e ; \mu\right) d t \leq \delta_{M}(\mu)\left\|u_{N}\right\|_{L^{2}(I ; V)}\|e\| \mathcal{Y} .
$$

We then use the identity

$$
\int_{I}\langle\dot{e}, e\rangle_{V^{\prime} V} d t=\frac{1}{2}\|e(T)\|_{H}^{2}-\frac{1}{2}\|e(0)\|_{H}^{2}
$$

together with the strong monotonicity condition (2) and the estimate above to derive the bound:

$$
\begin{aligned}
m_{a}(\mu)\|e\|_{\mathcal{Y}}^{2} & \leq \int_{I} a\left[u_{\delta}\right]\left(u_{\delta}, e ; \mu\right)-a\left[u_{N}\right]\left(u_{N}, e ; \mu\right) d t+\frac{1}{2}\|e(T)\|_{H}^{2} \\
=\int_{I}\langle\dot{e}, e\rangle_{V^{\prime} V} d t & +\int_{I} a\left[u_{\delta}\right]\left(u_{\delta}, e ; \mu\right)-a\left[u_{N}\right]\left(u_{N}, e ; \mu\right) d t+\frac{1}{2}\|e(0)\|_{H}^{2} \\
=\int_{I}\langle\dot{e}, e\rangle_{V^{\prime} V} d t & +\int_{I} a\left[u_{\delta}\right]\left(u_{\delta}, e ; \mu\right)-\tilde{a}\left[u_{N}\right]\left(u_{N}, e ; \mu\right) d t+\|e(0)\|_{H}^{2} \\
& +\int_{I} \tilde{a}\left[u_{N}\right]\left(u_{N}, e ; \mu\right)-a\left[u_{N}\right]\left(u_{N}, e ; \mu\right) d t \\
& \leq\|R(\cdot ; \mu)\|_{\mathcal{Y}_{\delta}^{\prime}}\|e\|_{\mathcal{Y}}+\delta_{M}(\mu)\left\|u_{N}\right\|_{L^{2}(I ; V)}\|e\|_{\mathcal{Y}},
\end{aligned}
$$

where we added and subtracted $\tilde{a}\left[u_{N}\right]\left(u_{N}, e ; \mu\right)$ to get the definition of the residual (30). Dividing both sides by $\|e\|_{\mathcal{Y}}$ yields the result.

We note that the assumption $u_{\delta}^{0} \in V_{N}$ implies that $\|e(0)\|_{H}=0$. We can guarantee this by choosing $\xi_{1}:=u_{\delta}^{0} /\left\|u_{\delta}^{0}\right\|_{V}$ as the initial basis for $V_{N}$ in the POD-Greedy procedure.

The computation of $\|R(\cdot ; \mu)\|_{\mathcal{Y}_{\delta}^{\prime}}$ requires the knowledge of its Riesz representer $v_{\delta, R}(\mu) \in \mathcal{Y}_{\delta}$. Thanks to the Riesz representation theorem, it can be obtained from the equation

$$
\left(v_{\delta, R}(\mu), v_{\delta}\right) \mathcal{Y}=R\left(v_{\delta} ; \mu\right) \quad \forall v_{\delta} \in \mathcal{Y}_{\delta} .
$$

Since the test space $\mathcal{Y}_{\delta}$ consists of piecewise constant polynomials in time, the problem (35) can be solved via the time-marching procedure for $k=1, \ldots, K$ as follows:

$$
\int_{I^{k}}\left\langle v_{\delta, R}(t ; \mu), v_{h}\right\rangle_{V} d t=\int_{I^{k}}\left\langle r(t ; \mu), v_{h}\right\rangle_{V^{\prime} V} d t \quad \forall v_{h} \in V_{h} .
$$


We note that $\hat{v}_{R}^{k}(\mu):=\left.v_{\delta, R}(\mu)\right|_{I^{k}}$ is constant in time; hence, the integration on the left-hand side of (36) is exact. For the right-hand side of (36), we represent $u_{N}(\mu) \in$ $\mathcal{X}_{\triangle t, N}$ as the linear function (11) on $I^{k}$ and use it as an input for the residual (30). We then apply the trapezoidal quadrature rule for the approximate evaluation of the integral. The quadrature rule is chosen such that the quadrature error is of the size of the error of the truth Crank-Nicolson solution. We thus need to solve the following problems:

$$
\left\langle v_{R}^{k}(\mu), v_{h}\right\rangle_{V}=R^{k}\left(v_{h} ; \mu\right) \quad \forall v_{h} \in V_{h}(k=1, \ldots, K),
$$

where $v_{R}^{k}(\mu)$ is our approximation of $\hat{v}_{R}^{k}(\mu)$ and the right-hand side is given by

$$
\begin{array}{r}
R^{k}\left(v_{h} ; \mu\right)=\frac{1}{2}\left[\left\langle g\left(t^{k} ; \mu\right)+g\left(t^{k-1} ; \mu\right), v_{h}\right\rangle_{V^{\prime} V}-\tilde{a}\left[u_{N}^{k}\right]\left(u_{N}^{k}, v_{h} ; \mu\right)\right. \\
\left.\quad-\tilde{a}\left[u_{N}^{k-1}\right]\left(u_{N}^{k-1}, v_{h} ; \mu\right)\right]-\frac{1}{\triangle t^{k}}\left\langle u_{N}^{k}-u_{N}^{k-1}, v_{h}\right\rangle_{H} .
\end{array}
$$

Therefore, the computation of the Riesz representer leads to a sequence of $K$ uncoupled spatial problems in $V_{h}$. The parameter separability structure of the residual

$$
R^{k}\left(v_{h} ; \mu\right)=\sum_{q=1}^{Q_{R}} \theta_{R, q}^{k}(\mu) R_{q}\left(v_{h}\right)
$$

is transferred by the linearity of the Riesz isomorphism to the parameter separability of its Riesz representer $v_{R}^{k}(\mu)$ together with the parameter dependent functions $\theta_{R, q}^{k}$ : $\mathcal{D} \rightarrow \mathbb{R}$. Therefore, for $1 \leq q \leq Q_{R}$, we have

$$
v_{R}^{k}(\mu)=\sum_{q=1}^{Q_{R}} \theta_{R, q}^{k}(\mu) v_{R, q} \text { with }\left(v_{R, q}, v_{h}\right)_{V}=R_{q}\left(v_{h}\right) \quad \forall v_{h} \in V_{h} .
$$

Finally, we state the formulas for the residual norm as well as the spatio-temporal norm of $u_{N}$. Since $\left.v_{\delta, R}(\mu)\right|_{I^{k}}$ is constant in time, the integration on $I^{k}$ is exact and we can compute the spatio-temporal norm of $v_{\delta, R}(\mu)$ as follows:

$$
\left\|v_{\delta, R}(\mu)\right\|_{\mathcal{Y}}^{2}=\sum_{k=1}^{K} \triangle t^{k}\left\|\hat{v}_{R}^{k}(\mu)\right\|_{V}^{2} \approx \sum_{k=1}^{K} \Delta t^{k} \Theta_{R}^{k}(\mu)^{T} \mathbf{G}_{R} \Theta_{R}^{k}(\mu),
$$

where $\mathbf{G}_{R}:=\left\{\left\langle v_{R, q}, v_{R, q^{\prime}}\right\rangle\right\}_{q, q^{\prime}=1}^{Q_{R}} \in \mathbb{R}^{Q_{R} \times Q_{R}}$ and $\Theta_{R}^{k}(\mu):=\left\{\theta_{R, q}^{k}(\mu)\right\}_{q=1}^{Q_{R}} \in \mathbb{R}^{Q_{R}}$. The isometry of the Riesz isomorphism implies that $\|R(\cdot ; \mu)\|_{\mathcal{Y}_{\delta}^{\prime}}=\left\|v_{\delta, R}(\mu)\right\| \mathcal{Y}$. Since $\left.u_{N}(\mu)\right|_{I^{k}}$ is a linear function in time, the trapezoidal quadrature rule on $I^{k}$ is exact. We then can compute the spatio-temporal norm $\left\|u_{N}\right\|_{\mathcal{Y}}$ of $u_{N} \in \mathcal{X}_{\triangle t, N}$ according to

$$
\begin{aligned}
\left\|u_{N}\right\|_{\mathcal{Y}}^{2} & \approx \sum_{k=1}^{K} \frac{\Delta t^{k}}{2}\left(\left\|u_{N}^{k}\right\|_{V}^{2}+\left\|u_{N}^{k-1}\right\|_{V}^{2}\right)+\left\|u_{N}^{0}\right\|_{H}^{2} \\
& =\sum_{k=1}^{K} \frac{\Delta t^{k}}{2}\left[\mathbf{u}_{N}^{k T} \mathbf{K}_{N} \mathbf{u}_{N}^{k}+\mathbf{u}_{N}^{k-1}{ }^{T} \mathbf{K}_{N} \mathbf{u}_{N}^{k-1}\right]+\mathbf{u}_{N}^{0 T} \mathbf{M}_{N} \mathbf{u}_{N}^{0},
\end{aligned}
$$


where $\mathbf{K}_{N}:=\left\{\left\langle\xi_{i}, \xi_{j}\right\rangle_{V}\right\}_{i, j=1}^{N} \in \mathbb{R}^{N \times N}$. Since in our case the reduced basis is orthonormal in $V, \mathbf{K}_{N}$ is the identity matrix. Despite of our quadrature rule exactness, the right-hand side of (40) serves as our approximation of $\left\|u_{N}\right\|_{\mathcal{Y}}^{2}$, since we use the solution of problem (22) in computing the norm of the reduced-basis problem (21). The operation count in the online phase, associated with computation of the residual norm and the spatio-temporal norm on $\mathcal{Y}$ is correspondingly $\mathcal{O}\left(Q_{R}^{2} K\right)$ and $\mathcal{O}\left(N K+N^{2}\right)$.

We note that our a posteriori error estimate takes into account the error of the non-linearity approximation (32). In our discrete time setting, it is approximated by a computable quantity

$$
\delta_{M}(\mu) \approx \max _{k \in K} \max _{x \in \Omega}\left|v_{M}\left(u_{N}^{k}(x) ; \mu\right)-v\left(u_{N}^{k}(x) ; \mu\right)\right| .
$$

Since the EIM approximation $v_{M}(\cdot ; \mu)$ is constructed out of truth solutions, we assume that $N$ is chosen in such a way that $v_{M}\left(u_{N}^{k}(x) ; \mu\right) \approx v_{M}\left(u_{\delta}^{k}(x) ; \mu\right)$. We note that (41) requires the knowledge of $v\left(u_{N}^{k}(\mu) ; x ; \mu\right)$ and thus one full evaluation of the non-linearity for all $K$ time steps on our finite-element mesh. Therefore, the certification procedure is not fully mesh-independent in the online phase and requires our mesh storage.

We note that we performed the series of numerical approximations in order to provide a computable bound for the right-hand side of (31). In particular, the residual (30) coincides with the residual (38) for the Crank-Nicolson time-marching scheme (12) after application of the trapezoidal quadrature rule. However, in order to invoke the definition of the residual (30), $u_{\delta}$ must be the solution to our reference problem (9). We also replace $\delta_{M}(\mu)$ by its computable surrogate (41). This finally gives

$$
\triangle_{N, M}(\mu) \approx \triangle_{N, M}^{c}(\mu)
$$

where $\triangle_{N, M}(\mu)$ is the computable. However, the bound in general is not rigorous, since we can not guarantee that $\triangle_{N, M}^{c}(\mu) \leq \triangle_{N, M}(\mu)$ holds.

\section{Examples and numerical results}

In this section, we consider examples of quasilinear parabolic PDEs with strongly monotone differential operators and apply the proposed reduced-basis techniques to these problems.

\subsection{D magnetoquasistatic problem: analysis}

For the first numerical example, we choose a 1D magnetoquasistatic approximation of Maxwell's equations (see, e.g. [2, 17]). Let $d=1, \Omega=(0,1)$ and $V:=$ $H_{0}^{1}(\Omega) \hookrightarrow L^{2}(\Omega)=: H$. The norm on $V$ is $\|u\|_{V}^{2}:=\left\langle u^{\prime}, u^{\prime}\right\rangle_{L^{2}}$, which is indeed a norm due to Poincare-Friedrichs inequality. We use the time interval $I=(0,0.2]$ 
and the parameter set $\mathcal{D}:=[1,5.5] \subset \mathbb{R}$. For a parameter $\mu \in \mathcal{D}$, we want to find $u:=u(\mu)$ which solves

$$
\begin{aligned}
\dot{u}-\left(v\left(\left|u^{\prime}\right| ; \mu\right) u^{\prime}\right)^{\prime}=g & \text { on } I \times \Omega, \\
u(t, x)=0 & \forall(t, x) \in I \times \partial \Omega, \\
u_{o}(x)=0 & \forall x \in \Omega .
\end{aligned}
$$

We here used $g(x, t):=12 \sin (2 \pi x) \sin (2 \pi t)$ and define $v(s ; \mu)=\exp \left(\mu s^{2}\right)+1$ as the reluctivity function.

We consider the quasilinear form for the weak formulation (6), which here is given by

$$
a[u](u, v ; \mu)=\int_{\Omega} v\left(\left|u^{\prime}\right| ; \mu\right) u^{\prime} v^{\prime} d x .
$$

If the function $v(\cdot ; \mu) \cdot: \mathbb{R}_{0}^{+} \rightarrow \mathbb{R}_{0}^{+}$is strongly monotone, i.e. if

$$
\left(v\left(s_{2} ; \mu\right) s_{2}-v\left(s_{1} ; \mu\right) s_{1}\right)\left(s_{2}-s_{1}\right) \geq m_{a}(\mu)\left(s_{2}-s_{1}\right), \quad \forall s_{2}, s_{1} \in \mathbb{R}_{0}^{+}
$$

holds, then (44) satisfies the strong monotonicity condition (2). Indeed, we set $s_{1}=$ $w^{\prime}, s_{2}=v^{\prime}$ and integrating we get

$$
\begin{aligned}
a[v](v, v-w)-a[w](w, v-w) & =\int_{\Omega}\left(v\left(v^{\prime} ; \mu\right) v^{\prime}-v\left(w^{\prime} ; \mu\right) v^{\prime}\right)\left(v^{\prime}-w^{\prime}\right) d x \\
\geq & m_{a}(\mu) \int_{\Omega}\left(v^{\prime}-w^{\prime}\right)^{2} d x=m_{a}(\mu)\|v-w\|_{V}^{2} .
\end{aligned}
$$

It is clear that the reluctivity function $v(s ; \mu)$ in our example satisfies (45). Furthermore, the monotonicity constant can be taken as $m_{a}=\inf _{\mu \in \mathcal{D}} \inf _{s \in \mathbb{R}_{+}} v(s ; \mu)$; hence, we have $m_{a}=2$ for our problem and the constant is parameter-independent. We also note that continuity of $v(\cdot ; \mu)$ implies hemicontinuity of (44) for every parameter $\mu \in \mathcal{D}$. Thus, the weak formulation (6) of the PDE (43) admits a unique solution.

We specify the bilinear form $\left\langle A^{\prime}(u ; \mu) v, w\right\rangle_{V^{\prime} V}=d a[u](v, w ; \mu)$ induced by the Fréchet derivative $A^{\prime}(u ; \mu): V \times \mathcal{D} \rightarrow V^{\prime}$ of the non-linear operator $A(u ; \mu)$. It is then used to compute the Jacobian matrix (15) for Newton method. In the present example, we have

$$
d a[u](v, w ; \mu)=\int_{\Omega}\left(2 \mu v^{\prime}\left(\left|u^{\prime}\right| ; \mu\right) u^{\prime}+v\left(\left|u^{\prime}\right| ; \mu\right)\right) v^{\prime} w^{\prime} d x .
$$

The derivative for the reduced-basis scheme in the formula (29), thanks to the chain rule, is given by

$$
\frac{\partial}{\partial u_{N, j}^{k}} v\left(\left|u_{N}^{\prime k}\left(x_{m}^{M}\right)\right| ; \mu\right)=2 \mu \nu^{\prime}\left(\left|u_{N}^{\prime k}\left(x_{m}^{M}\right)\right| ; \mu\right) u_{N}^{\prime k}\left(x_{m}^{M}\right) \xi_{j}^{\prime}\left(x_{m}^{M}\right),
$$

where all the indices are according to (29). In our numerical experiments, we drop the term $\mathbf{E}_{N, M}\left(\mathbf{u}_{N}^{k} ; \mu\right)$ in $\mathbf{A}_{N, M}^{\prime}\left(\mathbf{u}_{N}^{k} ; \mu\right)$. This then corresponds to an inexact Newtonlike method, which we use in our numerical experiments and which performed well. 


\subsection{D magnetoquasistatic problem: numerical results}

The truth approximation is performed by the Petrov-Galerkin scheme, which is introduced in Section 2, where $V_{h}$ is the finite element space, composed of piecewise linear and continuous functions, defined on the partition of $\bar{\Omega}$ into 100 equal subintervals and $\mathcal{N}_{h}=98$ nodes (excluding Dirichlet boundary nodes). For the time discretization, we divide the interval $I$ into $K=200$ subintervals of length $\Delta t=10^{-3}$. We solve the problem with the Crank-Nicolson scheme (12), while applying Newton's method, described in Section 2.2 on each time step for the numerical computation of the time snapshots. We iterate the Newton's method unless the norm of the residual (13) is less than the tolerance level, which we set to $10^{-8}$.

We generate the RB-EIM model as follows: we start from $\mathcal{D}_{\text {train }}^{\text {EIM }} \subset \mathcal{D}$ (a uniform grid of size 200) and compute truth solutions for each parameter in $\mathcal{D}_{\text {train }}^{E I M}$ to approximate the non-linearity $v$ with its EIM counterpart $v_{M}$. We set $M_{\max }=8$ as the maximal dimension of the EIM approximation space. Next, we run the POD-Greedy procedure with $M=M_{\max }$ and obtain $N_{\max }=5$ for $\varepsilon_{R B}=10^{-5}$, where $\mathcal{D}_{\text {train }}$ is a uniform grid over $\mathcal{D}$ of size 400 . For the POD-Greedy procedure and method certification, we use the computable bound $\triangle_{N, M}$ (42) for our error estimate $\triangle_{N, M}^{c}$ (31). We solve the problem with the reduced Crank-Nicolson scheme (22), while applying RB Newton's method, described in Section 3.2 on each time step for the numerical computation of the time snapshots. We iterate the Newton's method unless the norm of the residual (23) is less than the tolerance level, which we set to $10^{-8}$.

Next, we introduce a test sample $\mathcal{D}_{\text {test }} \subset \mathcal{D}$ of size 200 (uniformly random sample from $\mathcal{D}$ ) and the maximum of the estimator max $\triangle_{N, M}:=\max _{\mu \in \mathcal{D}_{\text {test }}} \triangle_{N, M}(\mu)$. We also introduce the following approximation of the "truth norm" error

$$
\varepsilon_{N, M}^{\text {true }}(\mu):=\left(\sum_{k=1}^{K} \frac{\Delta t^{k}}{2}\left(\left\|u_{\delta}^{k}(\mu)-u_{N}^{k}(\mu)\right\|_{V}^{2}+\left\|u_{\delta}^{k-1}(\mu)-u_{N}^{k-1}(\mu)\right\|_{V}^{2}\right)\right)^{1 / 2}
$$

and its maximum over the test sample $\max \varepsilon_{N, M}^{\text {true }}:=\max _{\mu \in \mathcal{D}_{\text {test }}} \varepsilon_{N, M}^{\text {true }}(\mu)$, where we use the Crank-Nicolson solutions $u_{\delta}^{k}(\mu)$ and $u_{N}^{k}(\mu)$ to compute (46). Hence, $\varepsilon_{N, M}^{\text {true }}(\mu)$ is only an estimate for $\|e(\mu)\|_{\mathcal{Y}}$ in (31), where the solution $u_{\delta}(\mu)$ of (9) and $u_{N}(\mu)$ of (21) enters. Once the reduced-basis model is constructed $\left(N_{\max }=7, M_{\max }=8\right)$, we verify the convergence with $N$ of $\max \triangle_{N, M}$ and $\max \varepsilon_{N, M}^{t r u e}$ on a test sample $\mathcal{D}_{\text {test }}$ and plot in Fig. 1 the $N-M$ convergence curves for different values of $M$. We can see that the estimator in Fig. $1 \mathrm{~b}$ reaches the desired tolerance level $\varepsilon_{R B}=10^{-5}$ for $\left(N_{\max }, M_{\max }\right)=(5,8)$.

Next, we investigate the influence of the EIM approximation error in the estimation process. We can split the bound (42) into two parts: the reduced-basis and the non-linearity approximation error estimation contributions

$$
\triangle_{N, M}^{R B}(\mu) \approx \frac{1}{m_{a}}\|R(\cdot ; \mu)\|_{\mathcal{Y}_{\delta}^{\prime}} \text { and } \triangle_{N, M}^{E I}(\mu) \approx \frac{\delta_{M}(\mu)}{m_{a}}\left\|u_{N}(\mu)\right\| \mathcal{Y}
$$




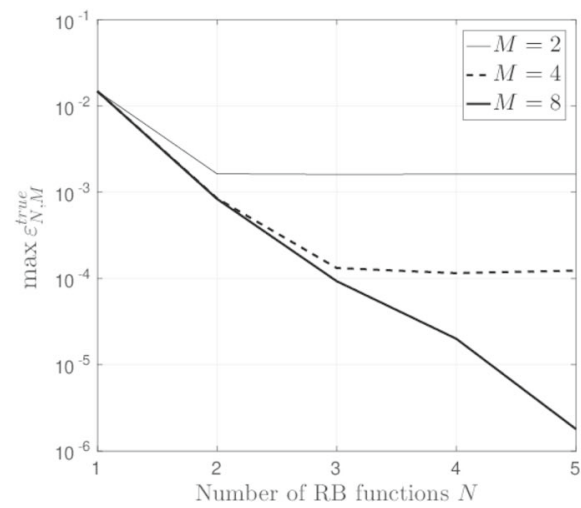

a

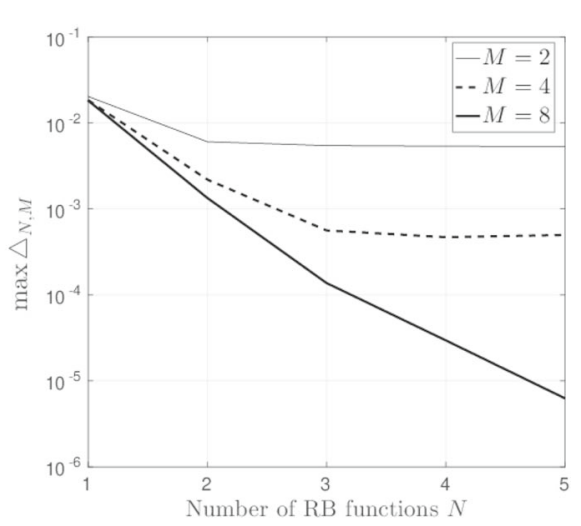

b

Fig. 1 a Convergence with $N$ of $\max \varepsilon_{N, M}^{\text {true }}$ for different values of $M$ on the test set, $1 \mathrm{D}$ example. b Convergence with $N$ of $\max \triangle_{N, M}$ for different values of $M$ on the test set, 1-D example

We compute these contributions as described in Section 2.2 and note that they serve only as computable surrogates for the respective contributions in our error estimate (31). We then set

$$
\triangle_{N, M}^{R B}:=\max _{\mu \in \mathcal{D}_{\text {test }}} \triangle_{N, M}^{R B}(\mu), \quad \triangle_{N, M}^{E I}:=\max _{\mu \in \mathcal{D}_{\text {test }}} \triangle_{N, M}^{E I}(\mu) .
$$

In Fig. 2a, we plot $\triangle_{N, M}^{R B}$ and $\triangle_{N, M}^{E I}$ for $1 \leq N \leq 5$ and $M=4, M=8$ : we can see that $M$ has nearly no influence on $\triangle_{N, M}^{R B}$, but we observe the "plateau" in $\triangle_{N, M}^{E I}$, which limits the convergence of the bound (42) with increasing $N$. The separation points, or "knees", of the $N-M$-convergence curves then reflect a (close-to) balanced contribution of both error terms.

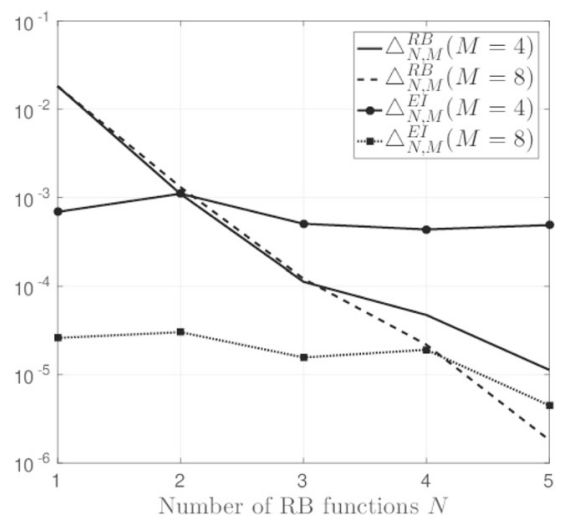

a

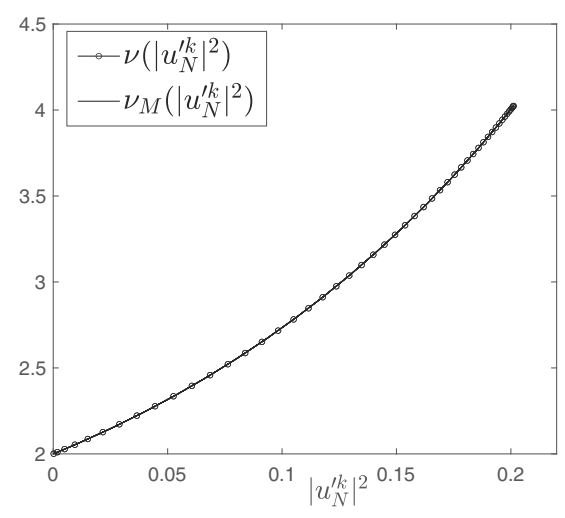

b

Fig. 2 a The dependence of $\triangle_{N, M}^{R B}$ and $\triangle_{N, M}^{E I}$ contributions with $N$ for fixed values of $M$. b: The reluctivity function $v\left(\left|u_{N}^{\prime k}\right|^{2} ; \mu\right)$ and its EI-approximation $(M=8) v_{M}\left(\left|u_{N}^{\prime k}\right|^{2} ; \mu\right)$ for the parameter $\mu=5.5$ at $t=0.2$ (b) 
Table 1 Performance of the 1D RB-EIM magnetoquasistatic approximation of Maxwell's equations on the test set

\begin{tabular}{lllllll}
\hline$N$ & $M$ & $\max \triangle_{N, M}$ & $\triangle_{N, M}^{R B}$ & $\triangle_{N, M}^{E I}$ & $\max \varepsilon_{N, M}^{\text {true }}$ & $\bar{\eta}_{N, M}$ \\
\hline 2 & 2 & $6.10 \mathrm{E}-03$ & $5.60 \mathrm{E}-03$ & $7.60 \mathrm{E}-04$ & $1.60 \mathrm{E}-03$ & 4.00 \\
3 & 4 & $5.62 \mathrm{E}-04$ & $5.05 \mathrm{E}-04$ & $1.12 \mathrm{E}-04$ & $1.32 \mathrm{E}-04$ & 5.82 \\
5 & 8 & $6.25 \mathrm{E}-06$ & $4.47 \mathrm{E}-06$ & $1.81 \mathrm{E}-06$ & $1.79 \mathrm{E}-06$ & 4.58 \\
\hline
\end{tabular}

In Table 1, we present, as a function of $N$ and $M$, the values of $\max \triangle_{N, M}$, $\triangle_{N, M}^{R B}, \triangle_{N, M}^{E I}, \varepsilon_{N, M}^{\text {true }}$ and mean effectivities $\bar{\eta}_{N, M}:=\frac{1}{\left|\mathcal{D}_{\text {test }}\right|} \sum_{\mu \in \mathcal{D}_{\text {test }}} \eta_{N, M}(\mu)$, where $\eta_{N, M}(\mu):=\triangle_{N, M}(\mu) /\left\|u_{\delta}(\mu)-u_{N}(\mu)\right\| \mathcal{Y}$. We note that the tabulated $(N, M)$ values correspond roughly to the "knees" of the $N$ - $M$-convergence curves. We can see that the effectivities are lower bounded by 1 and are of moderate size; thus, the bound (42) is reliable and there is no significant overestimation of our approximation of the "truth norm" error (46).

We then plot (see Fig. 2b) the reluctivity function $v\left(\left|u_{N}^{\prime k}\right| ; \mu\right)$ and its EI approximation $v_{M}\left(\left|u_{N}^{\prime k}\right| ; \mu\right)$ for the parameter $\mu=5.5$ at $t=0.2$; we can see that there is no visible difference between the original function and its EIM counterpart. Although the problem at hand is merely chosen to illustrate the methodology, we report on the average CPU time for comparison. The finite element method takes $\approx 0.47 \mathrm{~s}$ to obtain the solution, and the RB method $\left(N_{\max }, M_{\max }\right)$, which takes $\approx 0.08 / 0.10 \mathrm{~s}$ without and with the a-posteriori certification and results in the speed-up factor of $5.87 / 4.70^{1}$. We note that our implementation in the offline phase needs a large number of high-fidelity finite element solutions for the EIM approximation and the Riesz representers in (39). This implies large offline computational costs. However, these computations can be done in parallel to significantly reduce the time needed for the offline phase.

\subsection{D magnetoquasistatic problem: analysis}

As second example, we consider a 2D magnetoquasistatic problem for modelling of eddy currents in a steel pipe ${ }^{2}$. Let $\bar{\Omega}=\bar{\Omega}_{1} \cup \bar{\Omega}_{2}$ be a circular cross-section of the steel pipe with radius $r_{2}$, where $\Omega_{1}$ is the conducting domain (iron) and $\Omega_{2}$ is the non-conducting domain of radius $r_{1}$. The wire is represented by the part with the radius $r_{0}$ and the complementary part is the air gap (see Fig. 3a). We assume that the magnetic reluctivity function and the electric conductivity function have different structures on conducting and non-conducting domains, respectively, i.e.

$$
v(x, s)=\left\{\begin{array}{l}
v_{1}(s), \text { for } x \in \Omega_{1}, \\
v_{2}, \text { for } x \in \Omega_{2}
\end{array} \quad \text { and } \quad \sigma(x)=\left\{\begin{array}{l}
\sigma_{1}>0, \text { for } x \in \Omega_{1}, \\
\epsilon>0, \text { for } x \in \Omega_{2},
\end{array}\right.\right.
$$

\footnotetext{
${ }^{1}$ All the computations are performed in MATLAB on Intel Xeon(R) CPU E5-1650 v3, $3.5 \mathrm{GHz}$ x 12 cores, 64 GB RAM

${ }^{2}$ http://www.femm.info/wiki/TubeExample
} 
where $v_{2}, \sigma_{1}>0$ denote constants. We assume that the reluctivity function satisfies

$$
0<v_{\mathrm{LB}} \leq v(x, s) \leq v_{U B}, \quad \forall x \in \Omega, s \in \mathbb{R}_{0}^{+},
$$

where $v_{\mathrm{LB}}$ and $v_{U B}$ are accessible constants. We note that the air-gap and the coils in the steel pipe are electrically non-conductive, i.e. $\sigma(\xi)=0$ for $\xi \in \Omega_{2}$. However, we introduce a regularization parameter $\epsilon=10^{-8}$ as a value of $\sigma$ for the non-conducting domain. This allows us to consider a pure parabolic problem instead of a parabolicelliptic system with differential-algebraic structure (see, e.g. [13]). We set $\mu:=\sigma_{1}$ and define the parameter set $\mathcal{D}=\left[5 \cdot 10^{6}, 10^{7}\right]$ and the time interval $I=(0,0.02]$. We thus have a parametrized quasilinear parabolic equation

$$
\begin{aligned}
\sigma(x ; \mu) \dot{u}-\nabla \cdot(v(x,|\nabla u|) \nabla u) & =g & & \text { on } I \times \Omega, \\
u(t, x) & =0 & & \forall(t, x) \in I \times \partial \Omega, \\
u_{o}(x) & =0 & & \forall x \in \Omega .
\end{aligned}
$$

The right-hand side is the electric-flux density

$$
g(x, t)=\left\{\begin{array}{l}
\frac{I_{e}(t)}{2 \pi r_{0}}, \text { for } x \in \Omega_{1}, \\
0, \text { for } x \in \Omega_{2},
\end{array}\right.
$$

where $I_{e}(t)=100 \cdot \sin (100 \pi t)$ is the electric current.

We consider the quasilinear form for the weak formulation (6), which here is given by

$$
a[u](u, v ; \mu)=\int_{\Omega} v(x,|\nabla u| ; \mu) \nabla u \cdot \nabla v d x .
$$

In practical applications, the non-linear reluctivity function is often defined through magnetization curves or $|B|-|H|$ curves. The underlying physical properties of ferromagnetic materials determine the $|B|-|H|$ curve. These curves are naturally strongly monotone and, in practice, their analytical form is unknown. Instead, only a finite number of discrete points $\left(\left|H_{k}\right|,\left|B_{k}\right|\right), k=1, \ldots, K_{c}$ with $\left|H_{k}\right|,\left|B_{k}\right|$ denoting the magnitude of the magnetic field (measured in ampere/meter) and magnetic flux (measured in tesla), is given from the real life measurements. In order to reconstruct a continuous, monotone $|B|-|H|$ curve, monotonicity-preserving interpolation with cubic splines is applied [10]. We define a mapping $g_{1}: \mathbb{R}_{0}^{+} \rightarrow \mathbb{R}_{0}^{+}$which determines the magnetization curve via $|B|=g_{1}(|H|)$. An example of a $|B|-|H|$ curve, based on the measurements of a ferromagnetic material, which is used in our problem, is given in Fig. 3b; the real life measurements were provided by [18]. The mapping $s \mapsto v_{1}(s) s, s \in \mathbb{R}_{+}$then denotes the inverse $g_{1}^{-1}$ of $g_{1}$ and thus also is strongly monotone. The non-linear reluctivity function $v_{1}: \mathbb{R}_{0}^{+} \rightarrow \mathbb{R}_{0}^{+}$then is given by $v_{1}(s):=g_{1}^{-1}(s) / s, s \in \mathbb{R}_{+}$; it is required that $\nu_{1} \in C^{1}\left(\mathbb{R}_{0}^{+} ; \mathbb{R}^{+}\right)$and the spline approximation technique guarantees this property. If $g_{1}^{-1}(s)=v_{1}(s) s$ satisfies the strong monotonicity condition (45), then the mapping $\mathbf{S} \mapsto v_{1}(|\mathbf{s}|) \mathbf{s}, \mathbf{s} \in \mathbb{R}^{2}$ is strongly monotone with monotonicity constant $\nu_{L B}$ and Lipschitz continuous with Lipschitz constant $v_{U B}$. An example of magnetic reluctivity function $v_{1}(|B|)$ of ferromagnetic material used in our simulations is given in Fig. $3 \mathrm{c}$; here, we note that the magnitude of the magnetic flux $|B|$ corresponds to $|\nabla u|$ in our case. The mapping $\mathbf{S} \mapsto v_{2} \mathbf{S}, \mathbf{S} \in \mathbb{R}^{2}$ is linear; therefore, $\mathbf{s} \mapsto v(x,|\mathbf{s}|) \mathbf{s}$ is strongly monotone for all 


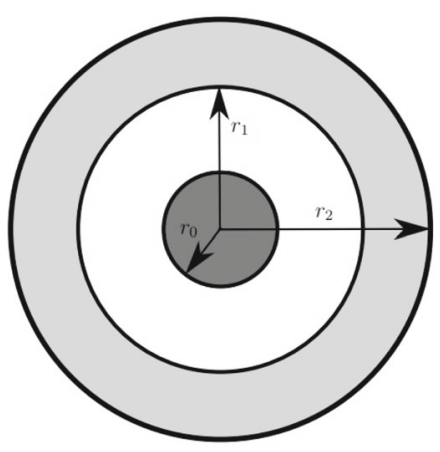

a

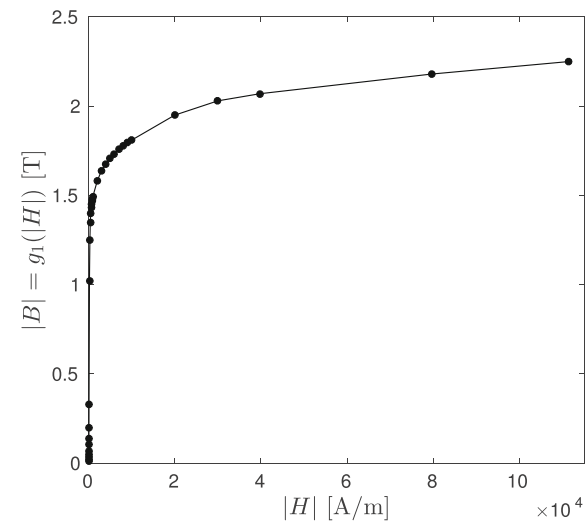

b

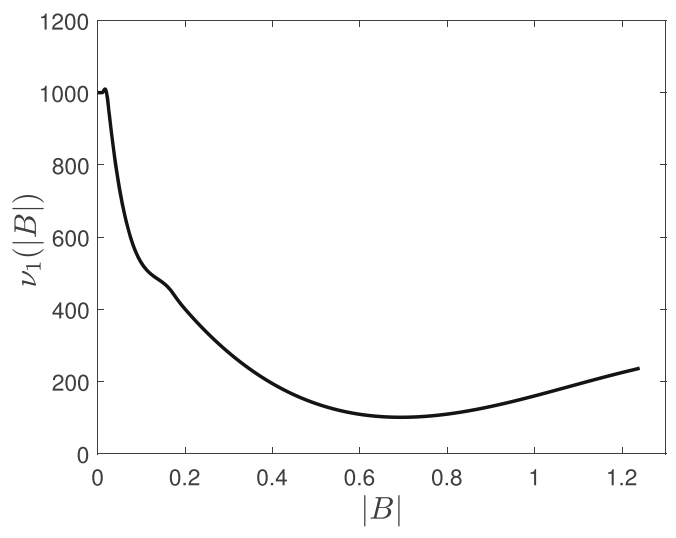

C

Fig. 3 a Geometry of the computational domain: the wire (dark grey), the air gap (white), the iron (bright grey). b An example of $|B|-|H|$ curve $g_{1}$ of ferromagnetic material used in our simulations (the measurements are denoted by dots). c An example of magnetic reluctivity function $v_{1}(|B|)$ of ferromagnetic material used in our simulations

$x \in \Omega$. The form (51) then is strongly monotone with the monotonicity constant $v_{L B}$ and Lipschitz continuous with the Lipschitz constant $3 v_{U B}$ (see [10] for the corresponding proofs). Hence, the weak formulation (6) of the PDE (50) admits a unique solution.

We specify the bilinear form $\left\langle A^{\prime}(u ; \mu) v, w\right\rangle_{V^{\prime} V}=d a[u](v, w ; \mu)$ induced by the Fréchet derivative $A^{\prime}(u ; \mu): V \times \mathcal{D} \rightarrow V^{\prime}$ of the non-linear operator $A(u ; \mu)$. It is then used to compute the Jacobian matrix (15) for Newton method. With

$$
n[u]=\left\{\begin{array}{l}
\frac{\nabla u}{|\nabla u|}, \text { for } \nabla u \neq 0 \\
0, \text { for } \nabla u=0
\end{array}\right.
$$


we have

$$
\begin{aligned}
& d a[u](v, w ; \mu)=\int_{\Omega} v^{\prime}(x,|\nabla u| ; \mu)(n[u] \cdot \nabla w)(\nabla u \cdot \nabla v) \\
& +v(x,|\nabla u| ; \mu) \nabla v \cdot \nabla w d x,
\end{aligned}
$$

and the derivative for the reduced-basis scheme in the formula (29), thanks to the chain rule, is given by

$$
\frac{\partial}{\partial u_{N, j}^{k}} v\left(x ;\left|\nabla u_{N}^{k}\left(x_{m}^{M}\right)\right| ; \mu\right):=v^{\prime}\left(x ;\left|\nabla u_{N}^{k}\left(x_{m}^{M}\right)\right| ; \mu\right) n\left[u_{N}^{k}\right]\left(x_{m}^{M}\right) \cdot \nabla \xi_{j}\left(x_{m}^{M}\right),
$$

where all the indices are according to (29).

In this example, the monotonicity constant $m_{a}(\mu)$ is not available analytically. As it was mentioned earlier in the discussion on $|B|-|H|$ curves, we can choose $v_{L B}>0$ as our monotonicity constant. However, since for each parameter $\mu \in \mathcal{D}$ there holds

$$
m_{a}(\mu):=\min _{k \in K} \min _{x \in \Omega} v_{1}\left(\left|\nabla u_{N}^{k}(x)\right| ; \mu\right) \geq v_{L B},
$$

and the computation of (52) only requires one full evaluation of the non-linearity, which already has been performed to evaluate (41), we here use $m_{a}(\mu)$ as our constant for the estimation.

\subsection{D magnetoquasistatic problem: numerical results}

The truth approximation is performed by the Petrov-Galerkin scheme, which is introduced in Section 2, where $V_{h}$ is the finite element space, composed of piecewise linear and continuous functions, defined on a triangle mesh containing 4374 triangles and $\mathcal{N}_{h}=2107$ nodes (excluding Dirichlet boundary nodes). For the time discretization, we divide the interval $I$ into $K=200$ subintervals of length $\triangle t=10^{-4}$. The non-linear reluctivity function $v_{1}$ is reconstructed from the real $B-H$ measurements using monotonicity-preserving cubic spline interpolation and $v_{2}$ value is chosen as the reluctivity of air. We then solve the problem with the Crank-Nicolson scheme (12), while applying Newton's method, described in Section 2.2, on each time step for the numerical computation of the time snapshots. We iterate the Newton's method unless the norm of the residual (13) is less than the tolerance level, which we set to $10^{-8}$.

We generate the RB-EIM model as follows: we start from $\mathcal{D}_{\text {train }}^{\text {EIM }} \subset \mathcal{D}$ (a uniform grid of size 200) and compute truth solutions for each parameter in $\mathcal{D}_{\text {train }}^{E I M}$ to approximate the non-linearity $v_{1}$ with the EIM counterpart $v_{1}^{M}$. We set $M_{\max }=44$ as the maximal dimension of the EIM approximation space. Next, we run the PODGreedy procedure with $M=M_{\max }$ and obtain $N_{\max }=14$ for $\varepsilon_{R B}=10^{-4}$, where $\mathcal{D}_{\text {train }}$ is a uniform grid over $\mathcal{D}$ of size 400 . For the POD-Greedy procedure and method certification, we use the computable bound $\triangle_{N, M}$ (42) for our error estimate $\triangle_{N, M}^{c}$ (31). The monotonicity constant is evaluated as in (52). We solve the problem with the reduced Crank-Nicolson scheme (22), while applying RB Newton's method, described in Section 3.2, on each time step for the numerical computation of the time snapshots. We iterate the Newton's method unless the norm of the residual (23) is less than the tolerance level, which we set to $10^{-8}$. 


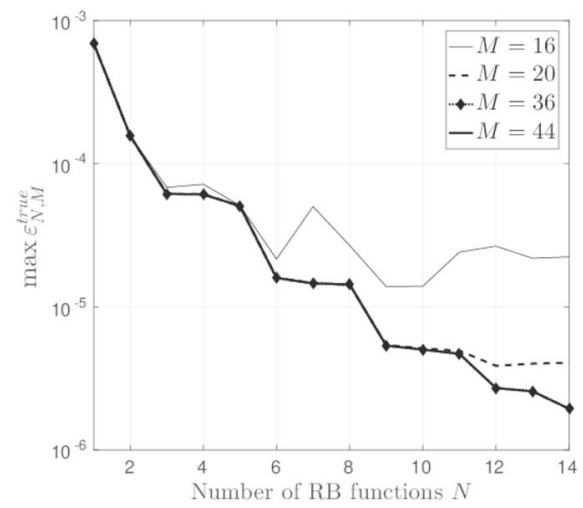

a

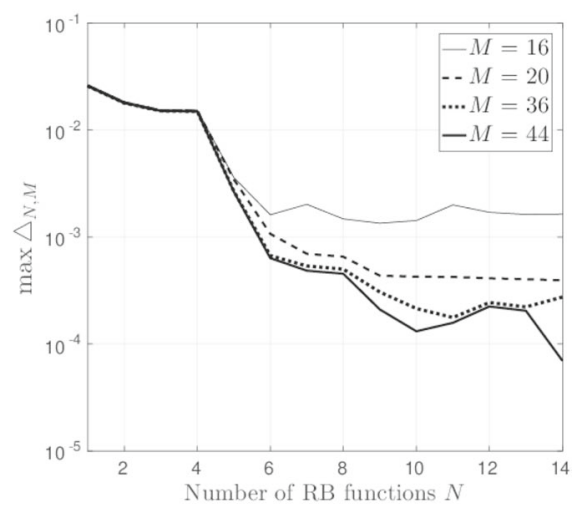

b

Fig. 4 a Convergence with $N$ of $\max \varepsilon_{N, M}^{\text {true }}$ for different values of $M$ on the test set, 2D example. b Convergence with $N$ of max $\triangle_{N, M}$ for different values of $M$ on the test set, 2D example

Then, we verify the convergence with $N$ of $\max \varepsilon_{N, M}^{\text {true }}$ (Fig. 4a) and $\max \triangle_{N, M}$ (Fig. 4b) on a test sample $\mathcal{D}_{\text {test }}$ (a uniformly random sample of size 200) for different values of $M$. We can see that the estimator in Fig. 3b reaches the desired tolerance level $\varepsilon_{R B}=10^{-4}$ for $\left(N_{\max }, M_{\max }\right)=(14,44)$. We note that the convergence is not monotone at some points due to the EIM interpolation of the non-polynomial non-linearity behind the problem. We can also see from Fig. 4a that increasing $M$ above 20 has nearly no impact on the convergence of the approximation of the "truth norm" error (46), but the bound (42) in Fig. 4b still shows a considerable decrease with increasing $M$. Indeed, in Fig. 5a, we plot $\triangle_{N, M}^{R B}$ and $\triangle_{N, M}^{E I}$ as defined in (48) for $1 \leq N \leq 14$ and $M=20, M=44$ : we can see that $M$ has nearly no influence on $\triangle_{N, M}^{R B}$, but we can observe the "plateau" in $\triangle_{N, M}^{E I}$, which limits the convergence of

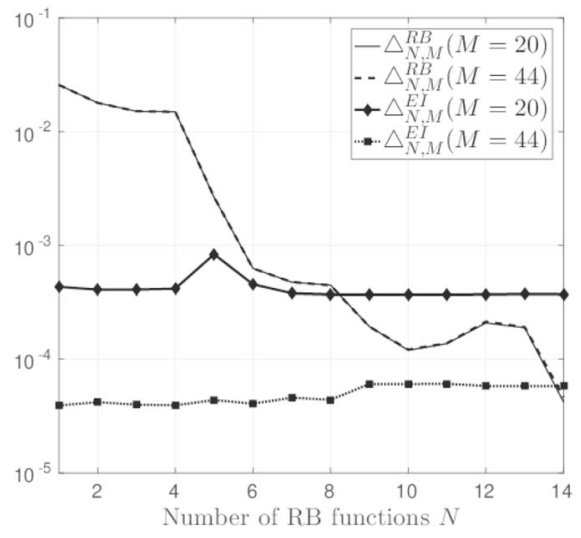

a

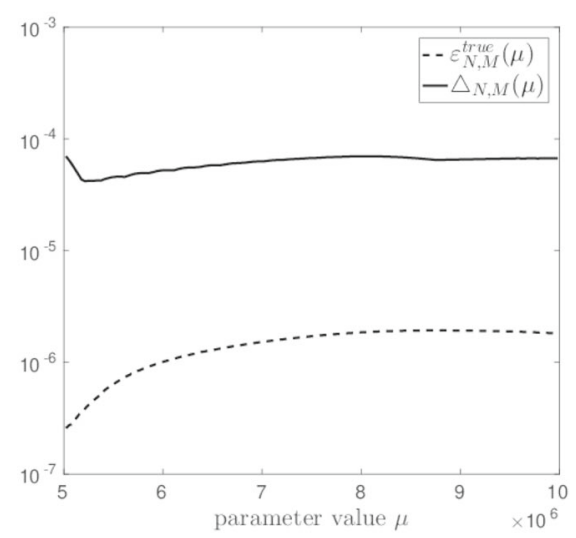

b

Fig. 5 a The dependence of $\triangle_{N, M}^{R B}$ and $\triangle_{N, M}^{E I}$ contributions with $N$ for fixed values of $M$. b Values of $\varepsilon_{N, M}^{\text {true }}$ and $\max \triangle_{N, M}$ for $\left(N_{\max }, M_{\max }\right)=(14,44)$ on the test set 
Table 2 Performance of 2-D RB-EIM model on the test set

\begin{tabular}{lllllll}
\hline$N$ & $M$ & $\max \triangle_{N, M}$ & $\triangle_{N, M}^{R B}$ & $\triangle_{N, M}^{E I}$ & $\max \varepsilon_{N, M}^{\text {true }}$ & $\bar{\eta}_{N, M}$ \\
\hline 6 & 16 & $1.60 \mathrm{E}-03$ & $6.68 \mathrm{E}-04$ & $1.40 \mathrm{E}-03$ & $2.15 \mathrm{E}-05$ & 98.06 \\
9 & 20 & $4.34 \mathrm{E}-04$ & $1.94 \mathrm{E}-04$ & $3.67 \mathrm{E}-04$ & $5.40 \mathrm{E}-06$ & 89.84 \\
11 & 36 & $1.76 \mathrm{E}-04$ & $1.38 \mathrm{E}-04$ & $1.04 \mathrm{E}-04$ & $4.68 \mathrm{E}-06$ & 64.28 \\
14 & 44 & $6.97 \mathrm{E}-05$ & $4.63 \mathrm{E}-05$ & $5.81 \mathrm{E}-05$ & $1.93 \mathrm{E}-06$ & 48.27 \\
\hline
\end{tabular}

the bound (42) with increasing $N$. We also plot the values of $\triangle_{N, M}(\mu)$ and $\varepsilon_{N, M}^{\text {true }}(\mu)$ and the error (46) for $\left(N_{\max }, M_{\max }\right)$ for every parameter $\mu \in \mathcal{D}_{\text {test }}$ in Fig. $5 \mathrm{~b}$.

In Table 2, we present, as a function of $N$ and $M$, the values of $\max \triangle_{N, M}$, $\triangle_{N, M}^{R B}, \triangle_{N, M}^{E I}$, max $\varepsilon_{N, M}^{t r u e}$ and the mean effectivities $\bar{\eta}_{N, M}$. We note that the tabulated $(N, M)$ values correspond roughly to the "knees" of the $N$ - $M$-convergence curves (see example 1 for the terminology and definitions). We can see that the effectivities are lower bounded by 1 , but the values are relatively large. Based on our experience with magnetostatics problems [11], we conject that this is related to the structure of the non-linearity and the effectivities are proportional to $C \cdot v_{U B} / v_{L B}$, where $C$ is some constant.

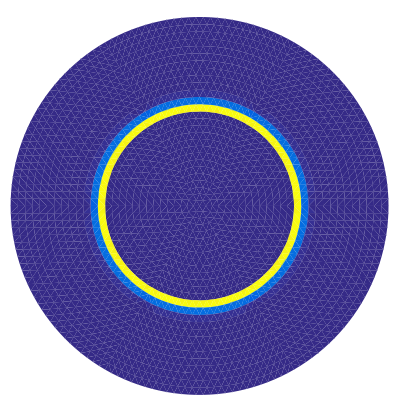

a

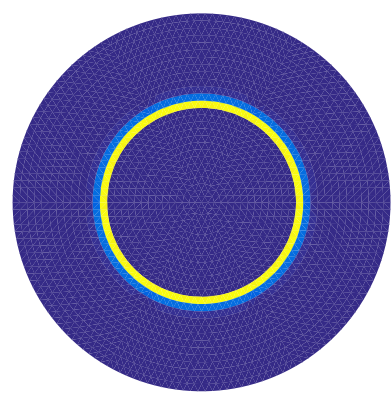

C
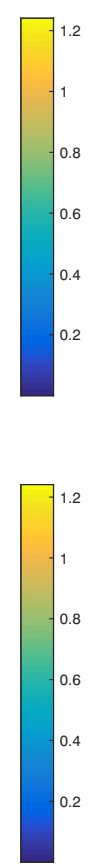

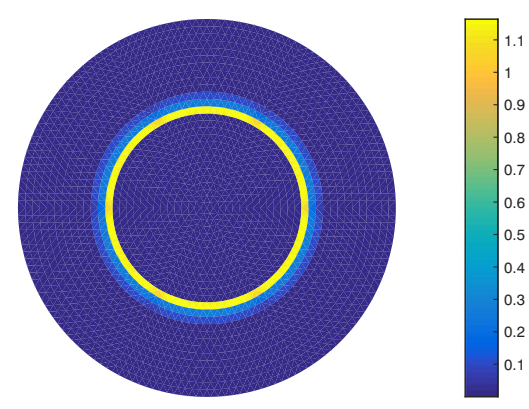

b

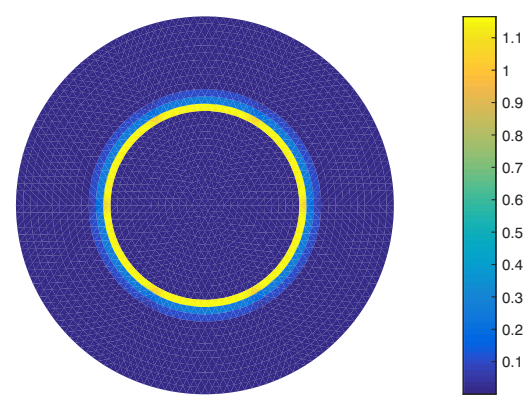

d

Fig. 6 The truth magnetic flux density $\left|\nabla u_{\delta}^{k}\right|$ for $\mu=10^{7}$ at $\mathbf{a} t=0.01, \mathbf{b} t=0.02$. The reduced-basis magnetic flux density $\left|\nabla u_{N}^{k}\right|$ for $\mu=10^{7}$ at $\mathbf{c} t=0.01, \mathbf{d} t=0.02$ 
In Fig. 6, we show the truth finite element magnetic flux density $\left|\nabla u_{\delta}^{k}(x, \mu)\right|$ and the corresponding reduced magnetic flux density $\left|\nabla u_{N}^{k}(x, \mu)\right|$ for $\mu=10^{7}$ and $t=$ 0.01 and $t=0.02$. We observe that flux densities look very similar. Next, we compare the average CPU time required for both the finite element method, which takes $\approx 70 \mathrm{~s}$ to obtain the solution, and the RB method with $\left(N_{\max }, M_{\max }\right)=(14,44)$, which takes $\approx 1.80 / 2.42$ without and with the a posteriori certification and results in the speed-up factors (rounded) of 39 and 29, respectively. The offline phase requires the knowledge of the truth finite-element solutions for the EIM approximation step. Since 200 truth solutions were generated in the consecutive order, it takes $\approx 4 \mathrm{~h}$. The generation of these truth solutions could be performed in parallel which would reduce the offline time. The POD-Greedy sampling takes $\approx 40 \mathrm{~min}$ for our implementation. We note that our implementation in the offline phase needs a large number of highfidelity finite element solutions for the EIM approximation and the Riesz representers in (39). This implies large offline computational costs. However, these computations can be done in parallel to significantly reduce the time needed for the offline phase.

\section{Conclusion}

In this paper, we propose the space-time reduced-basis method for quasilinear parabolic PDEs. We think that our space-time formulation combined with the chosen Petrov-Galerkin discretization provides an elegant approach to treat these kind of problems. We present a new a posteriori error estimate together with a computable bound, and we use it for the reduced basis construction with the POD-Greedy procedure. The developed methodology is applied to the magnetoquasistatic approximation of Maxwell's equations and numerical results confirm a good speed-up factor, which supports the validity of this approach. The reduced-basis methods developed in the paper will further be extended to treat more complicated industrial problems. It will further have a significant impact on the PASIROM project ${ }^{3}$, where the surrogate reduced-basis models are planned to be used in the optimization of electrical machines.

Funding Open Access funding enabled and organized by Projekt DEAL. Both authors acknowledge the support of the collaborative research project PASIROM funded by the German Federal Ministry of Education and Research (BMBF) under grant no. 05M2018.

Open Access This article is licensed under a Creative Commons Attribution 4.0 International License, which permits use, sharing, adaptation, distribution and reproduction in any medium or format, as long as you give appropriate credit to the original author(s) and the source, provide a link to the Creative Commons licence, and indicate if changes were made. The images or other third party material in this article are included in the article's Creative Commons licence, unless indicated otherwise in a credit line to the material. If material is not included in the article's Creative Commons licence and your intended use is not

\footnotetext{
${ }^{3}$ http://www.pasirom.de/
} 
permitted by statutory regulation or exceeds the permitted use, you will need to obtain permission directly from the copyright holder. To view a copy of this licence, visit http://creativecommons.org/licenses/by/4.0/.

\section{References}

1. Alla, A., Hinze, M., Kolvenbach, P., et al.: A certified model reduction approach for robust parameter optimization with PDE constraints. Adv. Comput. Math. 45, 1221-1250 (2019)

2. Bachinger, F., Langer, U., Schöberl, J.: Numerical analysis of nonlinear multiharmonic eddy current problems. Numer. Math. 100(4), 593-616 (2005)

3. Barrault, M., Maday, Y., Nguyen, N.C., Patera, A.T.: An empirical interpolation method: application to efficient reduced-basis discretization of partial differential equations. C.R. Acad. Sci. Paris Ser.I. 339(9), 667-672 (2004)

4. Glas, S., Mayerhofer, A., Urban, K.: Two ways to treat time in reduced basis methods. Model Reduct. Parametr. syst. 17, 1-16 (2017)

5. Grepl, M.A.: Certified reduced basis methods for nonaffine linear time-varying and nonlinear parabolic partial differential equations. Math. Models Methods Appl. Sci. 22(03), 1150015 (2012)

6. Grepl, M.A., Maday, Y., Nguyen, N.C., Patera, A.T.: Efficient reduced-basis treatment of nonaffine and nonlinear partial differential equations. ESAIM: Math. Model. and Numer. Anal. 41(3), 575-605 (2007)

7. Grepl, M.A., Patera, A.T.: A posteriori error bounds for reduced-basis approximations of parametrized parabolic partial differential equations. ESAIM: Math. Model. and Numer. Anal. 39(1), 157-181 (2005)

8. Haasdonk, B., Ohlberger, M.: Reduced basis method for finite volume approximations of parametrized linear evolution equations. ESAIM: Math. Model. and Numeric. Anal. 42(2), 277-302 (2008)

9. Haasdonk, B.: Reduced basis methods for parametrized PDEs - a tutorial introduction for stationary and snstationary problems. In: Benner, P., Cohen, A., Ohlberger, M., Willcox, K. (eds.) Chapter in model reduction and approximation: theory and algorithms, pp. 65-136. SIAM, Philadelphia (2017)

10. Heise, B.: Analysis of a fully discrete finite element method for a nonlinear magnetic field problem. SIAM J. Num. Anal. 31(3), 745-759 (1994)

11. Hinze, M., Korolev, D.: Reduced basis methods for quasilinear elliptic PDEs with applications to permanent magnet synchronous motors, Technical report: https://arxiv.org/pdf/2002.04288.pdf, (2020)

12. Ion, I.G., Bontinck, Z., Loukrezis, D., et al.: Robust shape optimization of electric devices based on deterministic optimization methods and finite-element analysis with affine parametrization and design elements. Electr. Eng. 100, 2635-2647 (2018)

13. Kerler-Back, J., Stykel, T.: Model reduction for linear and nonlinear magneto-quasistatic equations. Int. J. Numer. Methods Eng 111(13), 1274-1299 (2017)

14. Maday, Y., Nguyen, N.C., Patera, A.T., Pau, G.S.H.: A general multipurpose interpolation procedure: the magic points. Commun. Pure Appl. Anal. 8(1), 383-404 (2009)

15. Quarteroni, A., Manzoni, A., Negri, F.: Reduced basis methods for partial differential equations: an Introduction, vol. 92. Springer International Publishing, Switzerland (2016)

16. Rozza, G., Huynh, D.B.P., Patera, A.T.: Reduced basis approximation and a posteriori error estimation for affinely parametrized elliptic coercive partial differential equations. Arch. Comput. Methods Eng. 15(3), 229-275 (2008)

17. Salon, S.J.: Finite element analysis of electrical machines. Kluwer Academic Publishers, Boston (1995)

18. Schöps, S.: private communication (2020)

19. Steih, K., Urban, K.: Space-time reduced basis methods for time-periodic partial differential equations. IFAC Proc. Volumes 45(2), 710-715 (2012)

20. Urban, K., Patera, A.T.: An improved error bound for reduced basis approimation of linear parabolic problems. Math. Comp. 83(288), 1599-1615 (2014)

21. Volkwein, S.: Proper orthogonal decomposition: theory and reduced-order modelling, Lecture Notes, University of Konstanz (2013) 
22. Yano, M., Patera, A.T., Urban, K.: A space-time hp-interpolation-based certified reduced basis method for Burgers' equation. Math. Models Methods Appl. Sci. 24(09), 1903-1935 (2014)

23. Yano, M.: A space-time Petrov-Galerkin certified reduced basis method: application to the Boussinesq equations. SIAM J. Sci. Comput. 36(1), A232-A266 (2014)

24. Zeidler, E.: Nonlinear functional analysis and its applications i/a: linear monotone operators. Springer science + business media new york (1990)

25. Zeidler, E.: Nonlinear functional analysis and its applications II/b: nonlinear monotone operators. Springer science + business media new york (1990)

Publisher's note Springer Nature remains neutral with regard to jurisdictional claims in published maps and institutional affiliations. 